\title{
Transport of Large Scale Poloidal Flux in Black Hole Accretion
}

\author{
Kris Beckwith \\ Institute of Astronomy \\ University of Cambridge \\ Madingley Road \\ Cambridge CB3 OHA \\ United Kingdom \\ krcb@ast.cam.ac.uk \\ John F. Hawley \\ Astronomy Department \\ University of Virginia \\ P.O. Box 400325 \\ Charlottesville, VA 22904-4325 \\ jh8h@virginia.edu \\ and \\ Julian H. Krolik \\ Department of Physics and Astronomy \\ Johns Hopkins University \\ Baltimore, MD 21218 \\ jhk@pha.jhu.edu
}

\begin{abstract}
We report on a global, three-dimensional GRMHD simulation of an accretion torus embedded in a large scale vertical magnetic field orbiting a Schwarzschild black hole. This simulation investigates how a large scale vertical field evolves within a turbulent accretion disk and whether global magnetic field configurations suitable for launching jets and winds can develop. We find that a "coronal mechanism" of magnetic flux motion, which operates largely outside the disk body, dominates global flux evolution. In this mechanism, magnetic stresses driven by orbital shear create large-scale half-loops of magnetic field that stretch
\end{abstract}


radially inward and then reconnect, leading to discontinuous jumps in the location of magnetic flux. In contrast, little or no flux is brought in directly by accretion within the disk itself. The coronal mechanism establishes a dipole magnetic field in the evacuated funnel around the orbital axis with a field intensity regulated by a combination of the magnetic and gas pressures in the inner disk. These results prompt a reevaluation of previous descriptions of magnetic flux motion associated with accretion. Local pictures are undercut by the intrinsically global character of magnetic flux. Formulations in terms of an "effective viscosity" competing with an "effective resistivity" are undermined by the nonlinearity of of the magnetic dynamics and the fact that the same turbulence driving mass motion (traditionally identified as "viscosity") can alter magnetic topology.

Subject headings: accretion, accretion discs - relativity - (magnetohydrodynamics) MHD

\section{Introduction}

Astrophysical jets are seen in such a wide range of astrophysical systems that their creation must not require particularly unusual conditions. A tentative consensus has emerged that jets are a natural consequence of accretion, rotation and magnetic fields (for a summary of this position, see Livio 2000). Although the presence of accretion and rotation are a given in an accreting system, the presence of magnetic fields has long been considered to be less certain. However, since magnetohydrodynamic (MHD) turbulence driven by the magnetorotational instability (MRI; see Balbus \& Hawley 1991, 1998) accounts for the internal stresses that drive accretion, one is at least assured that the presence of accretion itself implies the existence of a tangled magnetic field of some reasonable strength.

But is that enough? Most jet launching mechanisms that have been developed depend on the existence of a large-scale, organized poloidal field, following in general terms the scenarios outlined analytically in the influential papers of Blandford \& Znajek (1977) and Blandford \& Payne (1982). In the Blandford-Payne model, a large-scale poloidal magnetic field is anchored in and rotates with the disk. If the fieldlines are angled outward sufficiently with respect to the disk, there can be a net outward force on the matter. As matter is accelerated along the rotating fieldlines, its angular momentum increases still further, accelerating and driving an outflow. In this model the power for the resulting jet comes from the rotation of the accretion disk. In the Blandford-Znajek model, the jet is powered by the rotating space-time of a black hole. (In the case of jets from systems with stars rather than black holes, stellar rotation can play a similar role.) Radial magnetic field lines lie along the hole's rotation 
axis and are anchored in the event horizon. Fieldline rotation is created by frame-dragging and this drives an out-going Poynting flux. Most simulations that have demonstrated jetlaunching have assumed the existence of a large-scale poloidal (mainly vertical) field in the initial conditions (see, e.g., the review by Pudritz et al. 2007). Although such simulations have provided considerable evidence that magnetic fields can be effective in powering jets, they cannot account for the origin of those fields.

If, as it seems, some sort of large-scale poloidal field is required for jet production, how can such a field be established in the near hole region? One possibility is an accretion disk dynamo, presumably working with the turbulent fields generated naturally by the MRI. A dynamo is an attractive possibility because it has the potential to be ubiquitous. A number of models have been put forward suggesting how some form of inverse cascade might occur within the MHD turbulence to generate a large scale field (e.g., Tout \& Pringle 1996 ; Uzdensky \& Goodman 2008), but these scenarios remain speculative and nothing definitive has been seen in global disk simulations done to date. In any case, the strict conservation of flux within a volume means that a disk dynamo could produce net flux only through interactions with boundaries, in this case either the black hole, or by expelling flux to large radius.

The global advection of net flux is an important process, therefore, whether the disk functions as a dynamo or net poloidal field is simply carried in to the near hole region from large radius. Even without any dynamo action, a weak net field might become locally strong it were concentrated by the accretion process. In the absence of a single field polarity at large distance, a near-hole net field could nevertheless be built up due to a random walk process as field is accreted (e.g., Thorne et al. 1986). Whether or not net field can be so accreted, however, is a matter of some uncertainty. The main concern is that the field would not be accreted if, as seems intuitively likely, the field intensity declines outward and the rate of diffusion of the field through the matter exceeds the rate at which matter accretes van Ballegooijen 1989; Lubow et al. 1994; Heyvaerts et al. 1996; Ogilvie \& Livio 2001). This competition between inward advection and outward diffusion is typically described in terms of an effective turbulent accretion viscosity $\nu_{t}$ determining the accretion rate, and an effective magnetic diffusivity, $\eta_{t}$ that sets the field diffusion rate. The fate of the field is then determined by the effective magnetic Prandtl number $\mathcal{P}_{m}=\nu_{t} / \eta_{t}$. In this picture, it is argued that the relevant scale for effective resistivity is $\sim H$ whereas the relevant scale for effective viscosity is $\sim R$; consequently, field can be brought inward only if $\mathcal{P}_{m}>R / H$.

It is far from clear, however, that field transport within MHD turbulence can be described in such terms. The picture just described assumes that the fluid motion is independent of the field, yet in Nature the dynamical coupling between fluid and field assures 
that even weak fields are amplified, and that the effective viscosity $\nu_{t}$ is, in fact, the result of magnetic stresses. Thus, there is no kinematic regime in which the Lorentz forces can be neglected. The resulting MRI-driven turbulence is inherently three-dimensional; indeed, three-dimensional studies are essential to generating long-term sustained MHD turbulence due to the fundamental restriction of the anti-dynamo theorem. By contrast, the framework of effective turbulent viscosity and diffusivity is cast in axisymmetry, in the hope that suitable time- and space-averaging of the field transport process in a fully turbulent, threedimensional disk can lead to some value of $\eta_{t}$ in much the same way that suitable averaging of correlations in the magnetic field, normalized to some suitable pressure term (either gas, magnetic or total pressure) leads to a value of $\nu_{t}$. But the primary mechanism by which magnetic fields change the fluid elements to which they are attached is through magnetic reconnection driven by the fluid turbulence, not gradual slippage via resistivity. The effective viscosity and magnetic diffusivity are derived from making the ansatz that stress and reconnection can be modeled as a viscosity or resistivity, i.e., a constant coefficient multiplying the gradient in orbital rotation rate or magnetic field strength. Whether or not such a model is appropriate to accretion flows remains to be determined. Finally, net flux and field topology are global concepts; a purely local description of field motion in terms of transport coefficients may not be sufficient.

Numerical simulations offer a promising approach to investigate these and other issues related to global field evolution within accretion flows. Of course, simulations entail their own set of difficulties: global simulations require adequately fine resolution over a wide range of radii in the disk body and throughout a good deal of the surrounding coronal region as well. The numerical grid boundaries should be sufficiently remote so as to minimize artificial effects on the disk. Although axisymmetric simulations can be of interest, the problem ultimately must be done in three dimensions because any axisymmetric calculation fails to describe properly essential properties of both the fluid turbulence and the magnetic field evolution. Finally, because we need to follow the disk long enough to observe net accretion in an approximate inflow equilibrium, the duration of the simulation must be long compared to typical local dynamical times in the disk.

Work along these lines is ongoing. Global, three dimensional black hole accretion simulations without initial fields threading the disk were carried out and described in a series of papers beginning with De Villiers et al. (2003). In these simulations initial weak dipole loops are contained within an isolated gas torus. The subsequent MRI-driven accretion flow carries the inner half of the initial field loop (with a single sign for the net vertical field component) down to the black hole, creating radial field as the loop is stretched. Differential rotation along that radial field leads to rapid growth of the toroidal field, especially within the plunging region of the flow. This growing toroidal field causes the ejection of a magnetic 
tower into the low density funnel region and the establishment of a global dipole field anchored in the black hole. This field can then produce a Poynting flux jet if the black hole is rotating.

Beckwith et al. (2008a) examined several alternative initial field configurations in comparison to the dipolar loop. Quadrupolar initial field loops produce a much weaker funnel field. A similar result was found by McKinney \& Gammie (2004) for axisymmetric simulations, and McKinney \& Blandford (2009) in a three-dimensional model. A variation proposed by Hawley \& Krolik (2006) and further elaborated upon by Beckwith et al. (2008a) is a series of dipolar loops contained within the accretion flow. Such loops can lead to the creation of a temporary net sense of vertical field that can support a significant field in a relativistic outflow along the rotation axis for a sizable period of time. Beckwith et al. (2008a) also computed a model that began with a purely toroidal field. In this case the MRI creates turbulence, field and angular momentum transport, but without creating any overall organization to the poloidal field. As a consequence, no funnel field is generated.

In this paper we continue our study of the influence of field topology on accretion and potential jet formation through a three-dimensional general relativistic MHD simulation with an initial field configuration consisting of a net vertical field passing through an orbiting ring of gas at modestly large radius. We will investigate the evolution of the disk in the presence of a net field while determining if such an initial condition can lead to the establishment of field configurations conducive to jet formation. From this study we hope to gain more insight into whether or not large scale fields can be advected along with the accreting gas and to lay the groundwork for further work.

The rest of this paper is structured as follows: In \$2, we describe the methodology and the initial conditions for our simulations. In $\$ 3$ we discuss their results as they pertain to magnetic flux motion. Finally, in $\$ 4$, we summarize our results, compare them with current models from the literature, and explain their implications for observations of relativistic jets in Nature.

\section{Numerical Details}

For this work we employ the general relativistic MHD code GRMHD developed in De Villiers \& Hawley (2003); De Villiers (2006). GRMHD has already been used in several studies to simulate black hole accretion in three spatial dimensions (De Villiers et al. 2003; Hirose et al. 2004; De Villiers et al. 2005; Krolik et al. 2005; Hawley \& Krolik 2006; Beckwith et al. 2008a. b). GRMHD solves the equations of ideal non-radiative MHD in the static Kerr 
metric of a rotating black hole using Boyer-Lindquist coordinates. Values are expressed in gravitational units $(G=M=c=1)$ with line element $d s^{2}=g_{t t} d t^{2}+2 g_{t \phi} d t d \phi+$ $g_{r r} d r^{2}+g_{\theta \theta} d \theta^{2}+g_{\phi \phi} d \phi^{2}$ and signature $(-,+,+,+)$. The relativistic fluid at each grid zone is described by its density $\rho$, specific internal energy $\epsilon, 4$-velocity $U^{\mu}$, and isotropic pressure $P$. The relativistic enthalpy is $h=1+\epsilon+P / \rho$. The pressure is related to $\rho$ and $\epsilon$ via the equation of state for an ideal gas, $P=\rho \epsilon(\Gamma-1)$. The magnetic field is described by two sets of variables. The first is the constrained transport (CT; Evans \& Hawley 1988) magnetic field $\mathcal{B}^{i}=[i j k] F_{j k}$, where $[i j k]$ is the completely anti-symmetric symbol, and $F_{j k}$ are the spatial components of the electromagnetic field strength tensor. From the CT magnetic field components, we derive the magnetic field four-vector, $(4 \pi)^{1 / 2} b^{\mu}=* F^{\mu \nu} U_{\nu}$, and the magnetic field scalar, $\left\|b^{2}\right\|=b^{\mu} b_{\mu}$. The electromagnetic component of the stress-energy tensor is $T_{(\mathrm{EM})}^{\mu \nu}=\frac{1}{2} g^{\mu \nu}\|b\|^{2}+U^{\mu} U^{\nu}\|b\|^{2}-b^{\mu} b^{\nu}$.

\subsection{Initial and Boundary Conditions}

In this study we carry out both axisymmetric and fully three dimensional simulations around a Schwarzschild black hole. The initial condition, as in previous works (De Villiers et al. 2003; Hirose et al. 2004; De Villiers et al. 2005; Krolik et al. 2005; Hawley \& Krolik 2006; Beckwith et al. 2008a b), consists of an isolated gas torus upon which a weak magnetic field is imposed. We use the torus model described by De Villiers et al. (2003) with an adiabatic index of $\Gamma=5 / 3$. The initial angular momentum distribution is slightly subKeplerian, with a specific angular momentum at the inner edge of the torus (located at $r=30 \mathrm{M}) \ell_{\text {in }} \equiv U_{\phi} / U_{t}=6.10$. For this choice of $\ell_{\text {in }}$, the pressure maximum is at $r \approx 40 M$ and the outer edge of the torus is located at $r \approx 60 M$. The angular period at the pressure maximum is $\Omega^{-1} \approx 250 \mathrm{M}$. With this inner edge radius, the resulting accretion flow should have sufficient radial range to permit a detailed study of the advection of vertical flux. The choice of angular momentum parameter at the inner edge of the torus, $\ell_{\text {in }}$, was made so that the initial torus (and hence the steady state accretion flow) would have a scale height similar to that of previous simulations (see e.g. Beckwith et al. 2008b). The torus is initially surrounded by zones filled with zero-velocity gas set to a constant value of density and pressure. The ratios of these "vacuum" values to the torus maximum are $2 \times 10^{-4}$ for the pressure, and $5 \times 10^{-6}$ for the density. Because the code is relativistic, the timestep is always limited by the time for light to cross the smallest cell; high Alfvén speeds in the low-density coronal region do not create any unique difficulty.

The initial magnetic field is homogeneous and vertical, filling the annular cylinder

$35 M \leq R \leq 55 M$ (where $R$ is the cylindrical radius). This configuration differs from 
the vertical field used in some previous studies (see e.g. Koide et al. 1999; McKinney \& Gammie 2004; De Villiers 2006) which fills all space, not just through the initial torus. By isolating the field in this manner, we know that any vertical flux that ends up crossing the black hole event horizon was brought in from field initially passing through the torus at large radius, rather than already being present at the horizon in the initial conditions. Also, because equilibrium field strengths scale strongly with radius, a global vertical field that is strong enough to be interesting at one radius is likely to be overwhelming at some radii while negligible at others. We prefer to see the global field strength emerge as an outcome of the evolution. In our configuration the initial vertical field is subthermal within the torus and is unstable to the MRI. It should be noted, though, that this initial field configuration is not in dynamic equilibrium outside of the torus. Also, this is but one very specific realization of a torus embedded in large scale field. Future experiments will need to explore a wider variety of initial configurations.

This initial field is computed from the curl of the four-vector potential, i.e.,

$$
F_{\alpha \beta}=\partial_{\alpha} A_{\beta}-\partial_{\beta} A_{\alpha}=A_{\beta, \alpha}-A_{\alpha, \beta}
$$

with only $A_{\phi} \neq 0$. The vector potential corresponding to a uniform (Newtonian) vertical field geometry is proportional to the cylindrical radius, i.e.,

$$
A_{\phi} \propto r \sin \theta
$$

The vector potential for the initial field is

$$
A_{\phi}=A_{0}\left\{\begin{array}{lc}
R_{\text {in }} & R<R_{\text {in }} \\
r \sin \theta & R_{\text {in }} \leq R \leq R_{\text {out }} \\
R_{\text {out }} & R>R_{\text {out }}
\end{array}\right.
$$

where $R_{\text {in }}$ and $R_{\text {out }}$ are $35 M$ and $55 M$. Outside of those cylindrical radii the field is zero. $A_{0}$ was specified so that the ratio of the average thermal to average magnetic pressure inside the torus is $\beta=100$. At the center of the torus $\beta=275$ and $\beta$ declines smoothly toward 1 near the edge of the torus. In the surrounding corona, $\beta=0.038$. The initial mass, field and $\beta$ distribution is shown in Figure 1 .

The simulation we will focus on is three-dimensional with $256 \times 256 \times 64$ zones in $(r, \theta, \phi)$. The simulation is labeled "VD0m3d." Given the challenge of adequately resolving such models and of doing anything like a convergence study, we also carried out two-dimensional simulations at two different resolutions. In two dimensions we used $256 \times 256$ and $512 \times 512$ zones in $(r, \theta)$. The two-dimensional simulations are labeled "VD0m2d" and "VD0h2d" where the "m" designates medium resolution and the " $\mathrm{h}$ " designates the higher resolution case. 
For all models the radial grid extends from an inner boundary at $r_{i n}=2.104$ to an outer boundary located at $r_{\text {out }}=500 \mathrm{M}$. The radial grid was graded using a logarithmic distribution that concentrates zones near the inner boundary. An outflow condition is applied at both the inner and outer radial boundaries. In contrast to the zero net flux simulations that we have performed previously, magnetic fields pierce the outer boundary in the initial state. This net flux is kept fixed throughout the simulation, but otherwise the field at the boundary can evolve. When the fluid velocity normal to the boundary is directed outward we perform a simple copy of fluid variables into the ghost zones that lie outside of the boundary. In the case where the fluid velocity is inward, the ghost zones are filled with a cold, low density, zero momentum fluid at the vacuum value so that only cold, very low density gas can enter the computational grid. To minimize the influence of the outer boundary it is located as far away from the region of interest as is feasible. Any torques that arise from the interaction of the outer boundary with the large scale vertical field should have minimal influence on the turbulent disk itself.

The $\theta$-grid spans the range $0.01 \pi \leq \theta \leq 0.99 \pi$. This creates a conical cutout that surrounds the coordinate axis. Such a cutout will prevent flow through the coordinate axis, but it is computationally advantageous to avoid the coordinate singularity. The size of this $\theta$-cutout is determined by two considerations. First, light travel times across narrow $\phi$-zones near the axis sets the timestep, so the cutout should not be too small. In this simulation, $\Delta t=2.53 \times 10^{-3} M$. The $\theta$ cutout must not be too large; initial vertical field should not cross the $\theta$-cutout before reaching the outer radial boundary at $r=500 M$. A reflecting boundary condition is used along the $\theta$-cutout. The $\theta$ zones were distributed logarithmically so as to concentrate zones around the equator.

The $\phi$-grid spans the quarter plane, $0 \leq \phi \leq \pi / 2$. The $\phi$ grid is uniform and periodic boundary conditions are applied. The use of this restricted angular domain significantly reduces the computational requirements of the simulation. Schnittman et al. (2006) examined the variance in surface density as a function of $\phi$ and found the characteristic size of perturbations to be around $25^{\circ}$. Thus, while some global features may be lost by using a restricted domain, the character of the local MRI turbulence is captured.

\subsection{Magnetic Flux}

In analyzing the evolution of the large scale poloidal flux we will make use of the poloidal flux function, which corresponds to the $\phi$-component of the magnetic vector potential (van Ballegooijen 1989; Heyvaerts et al. 1996; Reynolds et al. 2006), which we must calculate from the simulation data. In GRMHD, we directly evolve the components of the Faraday 
tensor, $F_{\mu \nu}$ using the alternative form of the induction equation (for further details see De Villiers \& Hawley 2003):

$$
\partial_{\delta} F_{\alpha \beta}+\partial_{\alpha} F_{\beta \delta}+\partial_{\beta} F_{\delta \alpha}=0
$$

The space-space components of $F_{\mu \nu}$ are identified with the constrained-transport (CT) magnetic fields via $\mathcal{B}^{i}=[i j k] F_{j k}$ :

$$
\mathcal{B}^{r}=F_{\phi \theta} ; \quad \mathcal{B}^{\theta}=F_{r \phi} ; \quad \mathcal{B}^{\phi}=F_{\theta r} .
$$

This identification allows us to write the induction equation in the familiar form

$$
\partial_{t} \mathcal{B}^{i}-\partial_{j}\left(V^{i} \mathcal{B}^{j}-\mathcal{B}^{i} V^{j}\right)=0,
$$

where $V^{i}=U^{i} / U^{t}$. The tensor $F_{\mu, \nu}$ is related to the magnetic induction in the fluid frame, $B^{\alpha}$ by the relation

$$
F_{\mu \nu}=\epsilon_{\alpha \beta \mu \nu} B^{\alpha} U^{\beta},
$$

where $\epsilon_{\mu \nu \delta \gamma}=\sqrt{-g}[\mu \nu \delta \gamma]$.

The Faraday tensor can also be written in terms of a vector potential:

$$
F_{\alpha \beta}=\partial_{\alpha} A_{\beta}-\partial_{\beta} A_{\alpha}=A_{\beta, \alpha}-A_{\alpha, \beta} .
$$

It is convenient to define an azimuthally-averaged poloidal flux function, $A_{\phi}$. This can be derived from the azimuthally-averaged CT field data by noting that the total (spatial) derivative of $A_{\phi}$ is

$$
d A_{\phi}=\frac{\partial A_{\phi}}{\partial r} d r+\frac{\partial A_{\phi}}{\partial \theta} d \theta .
$$

¿From the definition of the Faraday tensor, we have that

$$
F_{\phi \theta}=A_{\theta, \phi}-A_{\phi, \theta} ; \quad F_{r \phi}=A_{\phi, r}-A_{r, \phi} .
$$

After azimuthal averaging, this reduces to

$$
F_{\phi \theta}=-A_{\phi, \theta} ; \quad F_{r \phi}=A_{\phi, r} .
$$

The space-space components of $F_{\mu \nu}$ are identified with the $\mathrm{CT}$ magnetic fields via $\mathcal{B}^{i}=$ $[i j k] F_{j k}$ so that

$$
\mathcal{B}^{r}=F_{\phi \theta}=-A_{\phi, \theta} ; \quad \mathcal{B}^{\theta}=F_{r \phi}=A_{\phi, r} .
$$

We therefore have that

$$
d A_{\phi}=F_{r \phi} d r-F_{\phi \theta} d \theta=\mathcal{B}^{\theta} d r-\mathcal{B}^{r} d \theta .
$$


To find the flux from this differential, we could in principle integrate over any poloidal surface. For the radial motion of vertical flux through the disk, the surface of greatest significance is the equatorial plane. Because this surface is interrupted by the event horizon, we must stretch it over half of the event horizon. We will therefore define a flux function that is the sum of two parts: the radial flux through the inner horizon boundary,

$$
\left.\Psi(\theta)\right|_{r=r_{i n}}=\int_{0}^{\theta} d \theta^{\prime} \mathcal{B}^{r}\left(r_{i n}, \theta^{\prime}\right),
$$

and the vertical flux through the equator,

$$
\left.\Phi(r)\right|_{\theta=\pi / 2}=\int_{r_{i n}}^{r} d r^{\prime} \mathcal{B}^{\theta}\left(r^{\prime}, \theta=\pi / 2\right) .
$$

Using these functions, we can determine how the accumulated flux through the top half of the event horizon depends on polar angle, $\Psi(\theta)$, and how much total vertical flux has been brought to within radius $r$. The full flux function along the total path is

$$
\mathcal{A}(r, \theta=\pi / 2)=\Psi(\pi / 2)-\Phi(r) .
$$

This corresponds to the net flux piercing the surface covering the top hemisphere of the black hole horizon and the equatorial plane out to radius $r$. The full vector potential component $A_{\phi}(r, \theta)$ is obtained by integrating the $\mathrm{CT}$ variables throughout the computational domain starting from $r=r_{i n}$ and $\theta=0 . \mathcal{A}(r, \theta=\pi / 2)$ is therefore identical to $A_{\phi}(r, \theta=\pi / 2)$. For the three-dimensional simulation, VD0m3d, $\mathcal{B}^{r}$ and $\mathcal{B}^{\theta}$ are integrated over over the $\phi$ dimension prior to computing $A_{\phi}(r, \theta)$. Given the periodic nature of the $\phi$ direction this is sufficient to compute the total flux piercing the volume bounded by the equatorial plane and the black hole horizon. We note that this procedure is consistent with the approach adopted in the calculations of van Ballegooijen (1989), Heyvaerts et al. (1996), and Reynolds et al. (2006).

\section{Global Flux Transport}

\subsection{Overall evolution}

The simulation begins with an isolated torus threaded with a column of vertical field. The first part of the simulation is marked by relatively rapid evolution of the coronal field and the surface layers of the torus. Figure 2 shows the evolution of the poloidal magnetic flux and the density distribution over the time period $1000 M \leq t \leq 2500 M$. This process, along with the subsequent evolution of the flow is also shown in an animation included in the online 
edition of this work. We encourage the reader to refer to this material as it clarifies many of the issues that we shall discuss at length in the remainder of the text. As noted above, the initial state is not in equilibrium within the corona. There is differential rotation between the coronal gas and the torus, and the magnetic pressure within the vertical field column is not balanced by pressure within the initial atmosphere. The field expands radially, and stresses at the boundary of the disk begin to drive low density gas from the torus outward along field lines. In the inner half of the torus, low density surface layers move inward in two thin accretion streams above and below the equatorial plane, dragging poloidal field lines down to the black hole. This behavior was dubbed an "avalanche flow" when it was observed in the axisymmetric MHD accretion torus simulations of Matsumoto et al. (1996), and it is a common feature to accretion models that begin with vertical fields threading the disk (e.g., Koide et al. 1999). These surface features develop well before field deeper within the torus is significantly amplified by the MRI. The surface layers in the outer half of the torus also evolve, but here mass flows radially outward along the field lines, carrying the field along as it does so.

By $t=2000 M$ net flux has begun to build up on the black hole horizon, well before any significant mass accretion has begun. This flux arrives there by a process we call the "coronal mechanism," which we discuss briefly here and will discuss again in greater detail in $\S 3.2$. A typical field line participating in the coronal mechanism may be traced from the outer boundary at high latitude more or less vertically down to the upper surface of the torus, where it bends sharply toward the hole near the boundary of the denser region. It goes in some distance before doubling back (forming a "hairpin" shape) and returning to the torus. There it passes through the equator, and turns inward to mirror its behavior on the other side of the equator. A short time later, the apex of each hairpin moves inward and closer to the equator, where, in the early stages of this simulation when there is little mass at small radii, it can encounter an oppositely-oriented hairpin from the other hemisphere. Reconnection ensues, causing the magnetic flux distribution to change essentially instantaneously.

The four panels of Fig. 2 show these structures in differing ways. In the first panel, an isolated hairpin field line can be clearly seen approaching very close to the horizon. By the time of the second panel, the bends in this field line have reconnected, attaching it to the event horizon and liberating a pair of closed loops. Also in the second panel, new hairpins can be seen forming along the top and bottom surfaces of the disk in the region $20 M<r<30 M$. These hairpins evolve further and become embroiled in the beginnings of disk turbulence (the channel modes) in the third and fourth panels.

Magnetic field enters the extremely low-density funnel around the rotation axis by an offshoot of the coronal mechanism just described. There is initially no field within the 
funnel. Once horizontal field lines have been brought close to the black hole, their radial, near-horizontal components become unstable to the formation of ballooning half-loops that rise upward into the funnel. These loops are the initial source of field for the funnel. Only the inner half of the rising loop enters the funnel and, as a result, the field direction changes sign abruptly at the funnel's outer edge along the centrifugal barrier.

The coronal process also occurs at radii exterior to the initial pressure maximum of the torus. There it acts to move net flux progressively outward. Above the disk surface, the field is stretched out radially, but subsequently bends back down toward the equator, where reconnection can occur. This transfers the net flux from field lines going through the torus (those lines now close in large loops) to vertical field lines at large radius.

Thus, in the early phase of the evolution the global topology of the field is rapidly rearranged through coronal motions without any significant evolution within the main disk. Although these features result from the initial conditions, the process illustrates how (in principle) coherent large scale poloidal flux can be rapidly moved over large radial distances.

Although low density surface layers participate in the initial avalanche flow, the dense part of the torus is largely unaffected by these coronal motions. By $t=2500 M$, however, the MRI reaches a nonlinear amplitude throughout the torus and the characteristic structures associated with the "channel mode" have become visible within the torus. The next stage of evolution is illustrated in Figure 3 which shows snapshots every $500 M$ in time from 3000 to $4500 M$. Gas in the torus moves radially both inward and outward in the characteristic channels as angular momentum is transported along field lines, which are themselves increasingly stretched into long radial filaments. The channel modes are unstable to nonaxisymmetric perturbations and rapidly break up, resulting in a turbulent disk without prominent radial features. In the axisymmetric simulations, these relatively coherent large-scale radial flows continue to dominate throughout the evolution. This artificial persistence of coherent motion is a significant limitation of two-dimensional simulations with vertical field.

Accretion into the black hole does not begin until $1650 M$, and the mass accretion rate at the inner edge of the disk reaches its long-term mean for the first time shortly after $4500 \mathrm{M}$. The mass accretion rate then continues to vary by factors of $\sim 2$ around this mean through the remainder of the simulation. The disk achieves an approximate inflow equilibrium within $r \simeq 25 \mathrm{M}$ from time $10^{4} \mathrm{M}$ onward. Prior to $10^{4} M$, the mass in the inner disk $(r \leq 25 M)$ rises steadily, but after that time, it changes by about $10 \%$, and without any prevailing trend. The mass accretion rate averaged from $10^{4} M$ until the end of the simulation at $2 \times 10^{4} M$ is flat to within $10 \%$ for $r \leq 25 M$. The total mass on the grid at the end of the simulation is $78 \%$ of its initial value; of this, $18.8 \%$ has gone into the black hole. 
The divergence-free nature of the magnetic field means that the total flux is rigorously conserved except for losses at the boundaries of the computational domain. To measure the evolution of the total flux we integrate along the black hole horizon from the axis to the midplane and then out along the equator to the outer boundary. Figure 4 shows this integral as a function of time. The figure shows that there is no flux lost through the outer radial boundary during the simulation, but considerable net flux is deposited on the horizon at the expense of flux through the equator. The majority of the flux brought to the horizon arrives by $t \simeq 5000 M$, which implies that the net horizon field is created mostly by coronal motions, not through disk accretion. During the later stages of more or less steady-state accretion, the horizon flux grows much more slowly. Relative to its magnitude at $t=5000 M$, the horizon flux increases by only $28 \%$ over the next $5000 M$ and adds only another $11 \%$ during the last $10^{4} M$. By contrast, the total mass accreted onto the black hole grows by a factor of 9 from $5000 M$ to $2 \times 10^{4} M$, with a rate of increase that is approximately constant from $\simeq 10^{4} M$ onward.

The appearance of the overall flow at the end of the simulation is given in Figure 5 which shows the azimuthally-averaged density distribution and the $\beta$ parameter, and in Figure 6, which shows the vector potential component $A_{\phi}$ at time $t=2 \times 10^{4} \mathrm{M}$. There are several distinct regions. First, there is a low density, strongly magnetized funnel in which the field lines are primarily radial. Second, there is a corona characterized by large-scale field lines that loop about creating islands and hairpins, with numerous current sheets separating closely-spaced regions of oppositely-directed field. Here there are regions of both relatively strong and relatively weak magnetization; $\beta$ ranges from $\sim 0.1$ to $\sim 10$. A thin region of high $\beta$ fluid running along the funnel wall marks a current sheet between field lines directed in opposite radial directions as well as the boundary of the region with strong toroidal field. Finally, the main disk body remains weakly magnetized, with $\beta$ ranging from $\sim 10-100$. The disk thickness $H / R$ ranges from 0.1 to 0.15 , using the vertical density moment (Noble et al. 2008) for the definition of $H$. As can be seen from Figures 3 and 6, substantial parts of the disk body (most of the disk inside $r \simeq 40 M$ ) are actually disconnected from the large-scale field, their magnetic connections having been broken by the reconnection events that transferred field both to the horizon and to the outside of the torus. In the outer half of this region $(20 M \lesssim r \lesssim 40 M)$, however, some field lines do pass through the equator and out through the disk and into the corona.

This initial magnetic field and torus configuration was also simulated in axisymmetry. While the initial evolutions of the two- and three-dimensional models are similar, once the MRI sets in within the torus the subsequent evolutions differ dramatically. In axisymmetry, the channel modes dominate throughout the simulation, creating coherent radial field lines that extend over large distances. These field structures strongly influence the accretion 
of both mass and magnetic flux. Figure 7 compares the flux through the horizon, the mass accretion rate and the specific angular momentum accreted in simulations VD0m3d and VD0m2d. The axisymmetric simulation is characterized by very large fluctuations in

mass accretion rate. Similar large fluctuations are seen in the value of the magnetic flux through the horizon. Note the contrast between the net specific angular momentum $j_{\text {in }}$ carried into the hole by the accretion flow in the two- and three-dimensional simulations. For both plots the dashed line indicates the specific angular momentum of the innermost stable circular orbit at $6 \mathrm{M}$. The deep minima in the axisymmetric run correspond to strong torques created by radial field extending through the plunging region. These extended radial fields are associated with the coherent channel flows seen in axisymmetry, and they have an obvious strong effect on mass, angular momentum and magnetic fluxes carried into the black hole. The three-dimensional simulation is qualitatively different. In three dimensions, the channel modes quickly break up, yielding to genuine turbulence. The accretion rate into the hole fluctuates, but not nearly as strongly as in axisymmetry. Similarly, only in 3-d can one speak sensibly about a well-defined net accreted angular momentum per unit rest-mass, $j_{\text {in }}$. On the basis of these contrasts, we conclude that axisymmetric simulations, while useful for preliminary investigations of various initial configurations, are of only limited utility in investigating the long term behavior of MHD turbulent disks.

\subsection{The coronal mechanism}

The dominant mechanism for bringing flux to the event horizon in simulation VD0m3d is one that operates primarily in the corona, and not in the disk accretion flow proper. Although most of the flux is brought in during the first 5000M in time, there is a slow increase in flux throughout the remainder of the simulation. The majority of this flux appears to be transported through the continuing operation of the coronal mechanism. The key aspect is that magnetic field is carried inward by infalling low density fluid. Although this coronal mechanism operates outside the disk where most of the mass accretion happens, it, too, depends on angular momentum transport to proceed, and the basic physical mechanism for angular momentum transport is likewise the same: magnetic torques. Wherever the vertical field is perturbed so as to create a radial component, orbital shear acting on the radial field in turn creates toroidal field. Moreover, whenever the orbital frequency decreases outward, the relative signs of the radial and toroidal fields are always such as to make the $r$ - $\phi$ element of the magnetic stress tensor, $b^{r} b_{\phi} / 4 \pi$, negative. Such a stress transports angular momentum outward, tending to accentuate inflow, and therefore growth in the radial field component; this is, of course, the mechanism of the magneto-rotational instability. 
An example of the coronal mechanism in action is given in Figure 8. In this figure, color contours show the strength of the azimuthally averaged Maxwell stress per unit mass, overlaid with field lines derived from $A_{\phi}(r, \theta)$. For clarity we focus on those field lines that still pass through the equator in the accretion disk. The first frame, corresponding to $t=6000 M$, features a field that runs from the equator vertically upward through the disk and then down toward the hole before reversing and moving radially outward. The strong stress per unit mass in the vicinity of the hairpin carries angular momentum away from the associated matter, enabling it to move inward. In the next frame at $t=6200$, the hairpin moves in radially toward the black hole. The next two frames show a close up view of the innermost part of the accretion flow at times $t=6440$ and $6460 M$. By the time of the first one, the inner portion of the hairpin has reached and crossed the horizon. Only $20 M$ later, the inside hairpin has reconnected, forming a closed field loop. Thus, the net result of the coronal mechanism at this stage is to attach those fieldlines forming the exterior of the hairpin to the black hole. These fieldlines then extend out into the corona, ultimately attaching to the disk at much larger radius.

Although net local inflow of magnetic field is a necessary condition for flux accretion, it is not a sufficient one; the net flux changes only when reconnection occurs. Local motion alone is insufficient because magnetic flux is an essentially global concept, so local motion is not enough to determine net flux transport. This distinction is emphasized by contrasting the local magnetic inflow rate with global measures. We define the local rate by

$$
V_{\Phi}(r, \theta) \equiv \frac{1}{2 \pi} \int d \phi V^{r} \mathcal{B}^{\theta}
$$

averaged over the portion of the simulation during which the trapped flux grows slowly $\left(5000-2 \times 10^{4} M\right)$. This is shown in Figure 9, for which the radius was chosen to be $15 M$. As this figure shows, the local flux inflow speed is considerably greater just outside the funnel $(|\pi-\theta| \simeq 0.2 \pi)$, where it is $\simeq 0.5-1 \times 10^{-4}$, than in the midplane, where it is nearly two orders of magnitude smaller.

This local rate of flux inflow is, however, much larger than the global rate of flux accumulation. The global rate is $\partial A_{\phi} / \partial t$, which can be inferred from Figure 4 . For the purpose of this figure, the magnetic flux was separated into the flux piercing the midplane, $\Phi$, and the flux through the upper hemisphere of the black hole, $\Psi$. During the early stages of the simulation (before $5000 M), \partial \Psi / \partial t \simeq 1 \times 10^{-5}$, but this rate of increase diminishes at later times by at least an order of magnitude. Thus, the local inflow rate, $V_{\Phi}(\theta \simeq 0.2 \pi, 0.8 \pi) \simeq 1 \times 10^{-4}$ at $r=15 M$ during the latter $3 / 4$ of the simulation, is as much as two orders of magnitude greater than the net global rate. In other words, local field inflow alone does not suffice to create global flux inflow. 
Likewise, reconnection on its own is also a necessary condition for flux accretion, but not a sufficient one. In order for flux accretion to occur, reconnection must result in a global rearrangement of the field topology. This fact is illustrated by the field structures displayed in Figure 8. In that figure, a narrow current sheet lies between the two sides of the hairpin, permitting reconnection to happen there. In order to transfer magnetic flux inward through the accretion flow and increase the net flux in the funnel, reconnection must preferentially destroy the side of the magnetic hairpin that lies closest to the midplane. This is possible because reconnection can also occur across the equator, something that the funnel field cannot do. Figure 10 illustrates the idea. Coronal hairpin field loops bring field down to the inner disk, returning along the disk back out to large radius where they eventually pass through the equator and connect to another hairpin in the opposite hemisphere. The innermost bend of these hairpins can attach to the horizon. When this happens, the field appears to be almost a closed loop, with one end of the loop inside the horizon (e.g., the two inner-disk field lines in the gray region of the diagram). If the two oppositely-directed portions of one of these loops can meet at intermediate radius, the equatorial flux jumps inward. This inward jump drastically shortens the time until the entire field loop can accrete onto the black hole. When that happens, flux of one sign is removed from the horizon above the equator and flux of the other sign is removed from below the equator, leaving a net increase in flux piercing each horizon hemisphere.

An example of a reconnection event within VD0m3d that leads to this sort of global flux rearrangement is shown in Figure 11. In this figure, color contours denote the radial component of the CT-field, $\mathcal{B}^{r}$, indicating the direction of the fieldline, which are again overlaid with white contours derived from the azimuthally averaged $A_{\phi}(r, \theta)$. Initially $(t=$ $14600 \mathrm{M})$, fieldlines connect large radii to the black hole event horizon through the funnel region (deep blue region below midplane) and then proceed back out into the disk close to the midplane (yellow region below midplane). These fieldlines cross the midplane at large radius and proceed back to the black hole north of the midplane (light blue region north of midplane), before finally proceeding out to large radius through the funnel region (red region north of midplane). At time $t=14620 M$, the oppositely directly fieldlines that lie just above and below the midplane have reconnected at $r \sim 6 M$, and the resulting closed fieldloop (which is still attached to the black hole) is rapidly accreted. This process transfers flux from the outer disk to $r \sim 6 M$ (where the reconnection event occurs) and then down to the black hole as the field loop is accreted, arriving at the black hole with the outer edge of the loop.

The coronal mechanism resembles the flux advection mechanism proposed by Rothstein \& Lovelace (2008) in the sense that most flux transport takes place outside of the disk body, and that flux in the corona moves inward because magnetic stresses send angular momentum 
off to infinity. They emphasize the contrast between the turbulent disk and non-turbulent flow outside of the disk. We likewise observe that large-scale, relatively laminar flows within the low-density corona easily move field about; flux-freezing is an excellent approximation there. On the other hand, there are also several points of contrast between our results and the model of Rothstein \& Lovelace (2008). They argue that in the disk corona, defined as the region where $\beta \sim 1$, the $T_{\phi}^{z}$ component of the magnetic stress tensor could transport angular momentum outward as effectively as the $T_{\phi}^{R}$ component to which attention is more often directed, allowing the disk surface to move inward. Although this is similar to what we see in our simulation during the initial avalanche phase, it is different from the dynamics prevailing at later times. During the accretion phase of the simulation, the coronal field lines develop MRI-like bends at high altitudes above the disk $(>>H)$, so that the inflow occurs in the corona rather than along the disk surface. In addition, as shown by Figure 12 , which contrasts the azimuthal averages of $-b^{r} b_{\phi} / \rho$ and $-b^{\theta} b_{\phi} / \rho$, there is very little net angular momentum flux per unit mass in the polar angle direction except in the funnel (at latitudes not too far from the midplane, $\left.b^{\theta} b_{\phi} \simeq b^{z} b_{\phi}\right)$. Although the magnitude of $T_{\phi}^{\theta}$ is often comparable to $T_{\phi}^{r}$, it has very nearly the same probability of having either sign, so that its

mean value is small. On the funnel edge, $T_{\phi}^{\theta}$ is more likely to take angular momentum away from the disk but has magnitude on average somewhat smaller than $T_{\phi}^{r}$, while in the outer corona, on average it brings angular momentum toward the midplane.

\subsection{Inflow in the disk body}

The majority of the net flux on the black hole is brought there by the coronal mechanism during the initial $5000 M$ of time. However, the flux on the horizon does increase over the last $1.5 \times 10^{4} M$ and, while the coronal mechanism continues to operate during this interval, it is possible that part of the flux might be brought in through the accretion disk itself. As the right panel of Figure 9 makes plain, the local magnetic inflow rate $V_{\Phi}$ is roughly two orders of magnitude smaller in the midplane than it is at the funnel edge. Nonetheless, its magnitude is still consistent with the total flux inflow rate during the latter part of the simulation. It may therefore be possible for flux advection directly associated with the accretion flow in the disk body to account for some part of the flux accumulation during the quasi-steady accretion phase. In this section we attempt to estimate the size of that contribution.

We begin by comparing the rate of magnetic flux accretion to the rate of mass accretion. If magnetic flux and mass moved inward in lock-step, the functions

$$
\mathcal{M}(r, t) \equiv \int_{0}^{t} d t^{\prime} \dot{M}\left(r, t^{\prime}\right) / M_{0}
$$


and

$$
\mathcal{A}(r, t) \equiv A_{\phi}(r, t, \pi / 2) / A_{\phi}\left(r_{\max }, 0, \pi / 2\right)
$$

would be identical. These quantities are normalized to the initial mass, $M_{0}$, and the initial flux; $r_{\max }$ is the outer radius of the initial torus. In Figure 13 , we show the time-dependence of both these quantities at two fiducial radii, just outside the event horizon (left panel) and at $r=20 M$ (right panel). The former, of course, tells about the flux attached directly to the black hole and the mass deposited there. The difference between the two plots is the mass and flux within the accretion disk between the horizon and $r=20 M$; here we will refer to this region as the "inner disk."

In evaluating Figure 13, one must allow for an important distinction between the histories of mass and magnetic flux: the mass of the black hole must increase monotonically (and the mass of the inner disk, although not required to do so, generally does), but the net magnetic flux can increase or decrease, both by magnetic reconnection and by the radial motion of closed loops. Thus, the inflow of magnetic flux is highly intermittent, and the timederivative of flux within some radius can easily be negative. On short timescales, the ratio of the rate of flux accretion to mass accretion can vary by at least an order of magnitude, as well as fluctuate in sign. For this reason, a sensible comparison of the rate of magnetic flux inflow relative to that of mass can be made only in regard to long-term time averages. In addition, in order to evaluate the efficiency of this process in real disks, we must restrict these averages to times when the inner disk was in approximate inflow equilibrium: for this simulation, that means the latter half of the data, from $t=10^{4} M$ to $t=2 \times 10^{4} M$. Within this span, we smooth $\mathcal{A}(t)$ by taking a running average $1000 M$ wide and then compose the ratio of the change in $\mathcal{A}$ to $\mathcal{M}$ from $10^{4} M$ to $2 \times 10^{4} M$ using the smoothed data. We find that $\Delta \mathcal{A} / \Delta \mathcal{M} \simeq 0.47$ at the horizon, and $\simeq 0.50$ at $r=20 M$. In other words, relative to the initial amount embedded in the accretion flow, magnetic flux moves inward roughly half as fast as mass, and these rates are the same at both the horizon and $r=20 M$. If the mass is in a state of inflow equilibrium, the equality of these two ratios shows that the magnetic flux in the inner disk is also in equilibrium.

This ratio of inflow rates may be contrasted with the local ratio of magnetic flux to mass. Looking beyond the short-timescale fluctuations in the plots of $\mathcal{A}(t)$ in Figure 13 , one sees that the average values of the flux at the horizon and the flux contained within $r=20 M$ are very similar. In other words, the vertical magnetic flux through the inner disk, $\Phi(r=20 M)$, contributes very little to $\mathcal{A}$. Time-averaging over the last $10^{4} M$ of data, we find that it is only $\simeq 0.86 \%$ of the initial flux, and it appears that what flux there is is confined to the outer part of the inner disk. By contrast, averaged over the same period, $5 \%$ of the initial mass can be found in the inner disk. Thus, in the inner disk, the time-averaged flux/mass ratio is only $17 \%$ of the initial value. 
To reconcile this flux/mass ratio with the accreted flux/mass ratio, there are only two possibilities: either the magnetic flux moves in at the same rate (or slower) than the mass and (at least) $2 / 3$ of the flux reaching the horizon between $10^{4} M$ and $2 \times 10^{4} M$ arrived there via a different route (most likely the coronal mechanism), or the magnetic flux on average moved inward three times faster than the mass. We view the former alternative as much more plausible. Note, however, any flux delivery from outside the inner disk must be balanced by flux losses from the inner disk to the horizon because the magnetic inflow rate at $r=20 M$ is nearly the same as at the horizon.

Next we further explore the nature of the magnetic flux equilibrium in the inner disk, using two different views of this quantity. Figure 14 shows the distribution of the vertical flux function $\Phi(r)$ time-averaged over the last $5000 M$ of the simulation and normalized to the initial flux value. For comparison, we also plot in that figure both the initial flux distribution and the final mass distribution in the disk, both likewise normalized to the initial total. The radial derivative $d \Phi / d r$ shows the magnitude and sign of the vertical magnetic field piercing the equator. We see that, like the mass, vertical flux has spread away from its initial location, both in and out, but, as already mentioned, a much smaller part of the net magnetic flux than the mass resides in the inner disk.

The time-dependence of the magnetic flux in the inner disk over the last $10^{4} M$ in time is illustrated in Figure 15. As this figure shows, not only is the magnetic flux contained within the inner disk a rather small part of the total, it fluctuates in time, frequently going negative. Little sign of any long-term trend can be seen, consistent with our contention that the inner disk magnetic flux has reached a statistical equilibrium. The frequent sign changes of the local net flux suggest that the poloidal magnetic field lines in the inner disk predominantly close within the inner disk.

Moreover, the magnetic field corresponding to the net flux is a very small fraction of the total field within the disk. Figure 16 shows the azimuthally averaged value of $\mathcal{B}^{\theta}$ through the equator at the end time as a function of radius, along with the initial value. The MRI has generated considerable field of both signs throughout the disk. Local regions of the disk have a net vertical flux, but their contrasting signs lead to little contribution to the total disk flux. The global net flux is present only as a slight positive excess moving both inward and outward with time.

To reach the state described by these figures, most of the large-scale magnetic connections to the disk matter must have been reconnected away. But this should come as no surprise because we have already seen that a significant part of the original flux has moved by reconnection from the disk to the horizon. The matter in the inner disk has little net flux because it has been largely bypassed through the coronal mechanism. Thus, while the 
MRI has created a large effective turbulent viscosity in the sense that considerable mass has accreted from the location of the initial torus, very little net flux has moved with it. In other words, within the disk, turbulence created by the MRI transports angular momentum rapidly and drives accretion, but very little flux moves with the accreting mass.

\subsection{Strength of the Funnel Field}

Lastly, we raise the question of whether in the very long-term the magnetic flux on the horizon would continue to grow. If it is regulated by a combination of the magnetic and gas pressure near $r=6 M$, and these are in an approximately time-steady state, one might expect that further flux accumulation on the horizon would have to stop. Figure 17 supports this idea. This figure shows that the magnetic pressure inside the funnel, the magnetic pressure in the inner accretion flow, and the gas pressure in the inner accretion flow are well correlated with one another in a temporal sense, even while all three change by more than two orders of magnitude over the course of the simulation. The time-dependent data show fluctuations that are so large, however, that the much slower accumulation of flux seen in Figure 13 is well below the noise, so our data on magnetic and gas pressures cannot answer any questions about long-term saturation of magnetic flux attached to the horizon.

That the magnetic and gas pressures should be closely related is an old idea (Rees et al. 1982; MacDonald \& Thorne 1982), but there is also a long history of controversy about whether the magnetic field pressure in the funnel should be closer in magnitude to the (poloidal) magnetic pressure or the gas pressure in the inner disk. For example, Ghosh \& Abramowicz (1997) and Livio et al. (1999) argued that as the poloidal magnetic field strength in the disk is subthermal, the funnel field strength should be regulated by it rather than the gas pressure. In the simulation presented here, the total, i.e. poloidal plus toroidal magnetic pressure in the disk is smaller than the gas pressure for the duration of the simulation (i.e. the field remains subthermal), and the magnetic pressure from the field in the funnel lies between these two candidate regulators, sometimes closer to one and sometimes closer to the other. Thus, the funnel field is consistently stronger than the poloidal field in the disk.

The relevance of the inner disk poloidal field to the strength of the funnel field seems to be rather limited based upon what we observe about the process carrying flux to the horizon. The field in the funnel is nearly radial, and its total intensity is determined by the poloidal flux that is delivered to the black hole over the course of the simulation. This flux delivery system is the coronal mechanism, and so it should not be surprising that the poloidal flux within the accretion disk itself plays at most a secondary role (e.g., by determining the rate of reconnection and accretion of flux loops). Instead, simple pressure-matching in the 
inner regions of the accretion flow-be it gas, (total, mostly toroidal) magnetic or radiation pressure - appears to be important in regulating how much flux may be attached to the black hole. We speculate that when the flux on the horizon has a total field intensity larger than would be consistent with the gas and magnetic pressure in the nearby accretion flow, the rate of reconnection along the funnel field boundary rises, so that the field approaching the black hole is entirely in closed loops and does not add to the net flux on the horizon.

\section{Summary, Discussion \& Conclusions}

In this paper we investigate the evolution of an accretion torus embedded in a large-scale vertical magnetic field orbiting a Schwarzschild black hole, with a view toward studying how magnetic flux moves relative to the accretion flow. The simulation stretched over $2.0 \times 10^{4} \mathrm{M}$ in time, corresponding to $80 \Omega^{-1}$ at the initial torus pressure maximum, long enough to establish inflow equilibrium in the inner disk for the second half of the simulation. Of particular interest is how the net vertical field evolves, and whether or not a field distribution consistent with the formation of jets or winds can develop. We trace the evolution of the net poloidal flux distribution with a particular focus on net flux that becomes attached to the black hole. Our primary result is that a significant fraction of the initial flux $\simeq 27 \%$-is brought to the black hole horizon, even though only a rather smaller fraction of the initial mass $\simeq 19 \%$ is accreted. The flux attached to the horizon supports a coherent poloidal field within the evacuated axial funnel, a requirement for the creation of a Blandford-Znajek type jet (if the black hole rotates, which it did not in this simulation). The mass and flux are carried inward through distinct mechanisms: the mass by turbulent stresses within the accretion disk, and the flux by large-scale motions in the low density corona.

Most of the global magnetic flux motion is mediated by a novel mechanism that we have dubbed the coronal mechanism. Rather than the gradual "diffusive" process that had been the focus of most previous discussions of magnetic flux motion in accretion flows, in this mechanism the flux is brought directly down to the horizon as the net flux jumps discontinuously when large-scale magnetic loops reconnect across the equator. The same orbital shear that creates angular momentum transport in the disk body by correlating radial and azimuthal magnetic field also acts in the corona; the difference is that in the corona the resulting stress leads to the formation of large-scale loops stretched rapidly inward rather than to MRI-driven turbulence. These stretched loops can reconnect to oppositely-directed field at much smaller radius than their footpoint, leading to sudden macroscopic radial changes in the location of magnetic flux. Although the coronal mechanism acts particularly rapidly during the initial transient phase of the simulation, it continues to dominate flux 
motion throughout the simulation, including the long period during which the inner disk is in approximate inflow equilibrium. We might therefore reasonably expect it to be a property of actual accretion disks.

The same reconnection that creates global relocation of net flux simultaneously creates closed field loops within the disk body. For this reason, the flux/mass ratio within the accretion flow is suppressed by a factor of order unity relative to what it is in the initial state. Earlier global disk simulations have, in most cases, assumed zero net flux for reasons of simplicity and computational convenience; the coronal mechanism might, in fact, make this assumption a reasonable approximation to the magnetic field in typical disks. The suppression of flux/mass in the disk body also makes conventional advection of flux in direction association with mass accretion relatively inefficient.

As seen in previous simulations (particularly Beckwith et al. 2008a), if the overall field topology at the black hole is dipolar, the funnel field can be relatively immune to reconnection and hence long-lived. We conjecture that the funnel field amplitude is determined by pressure balance with the gas and (total, mostly toroidal) magnetic pressures in the inner disk and coronal regions. Future experiments could test this hypothesis by running much longer simulations with more available net field to see if the field strength levels off or continues to build.

As with any simulation, our results are subject to some uncertainty due to the limitations inherent in a numerical solution. One concern is the importance of reconnection to the overall flux evolution. In our numerical simulation, reconnection occurs at the grid scale when oppositely signed field components are brought together. Although leaving the microscopic rate of such an important process to numerical effects is a concern, the overall motions that lead to the formation of current sheets and subsequent reconnection area driven by events on much larger scales. In this sense, we might argue that the rate of reconnection seen in the simulation is relatively independent of our gridscale. We have not, however, demonstrated that our results are numerically converged, although the global field motions in the axisymmetric simulations carried out at two different resolutions were very similar. Furthermore, the thermodynamics of these simulations are incomplete because energy lost to numerical magnetic reconnection and numerical cancellation of fluid velocities is not captured; conversely, we do not account for radiation in any way, either as a cooling agent or as a contributor to the pressure. One thing that is clear, regardless of any other numerical limitation, is that axisymmetric simulations are of limited utility. In addition to the constraints associated with the anti-dynamo theorem, the vertical field channel modes remain dominant throughout the simulation, giving a qualitatively distinct evolution at late time. Three dimensional simulations are essential for studying long term, steady-state behavior. 
To place our somewhat surprising results in context, it is worthwhile contrasting them with previous suggestions about how magnetic flux moves through accretion flows. These are quite disparate, for they have in general been based on approximate analytic arguments. We examine three principal approaches, of which one has received much more attention than the others.

The greatest amount of attention has been given to a picture in which the matter moves inward by an unspecified angular momentum transport process (called "viscosity", but not thought to be literally that) while the magnetic field diffuses relative to the matter through another mechanism called "turbulent resistivity", but similarly unattached to specific microphysics (e.g., van Ballegooijen 1989; Lubow et al. 1994; Heyvaerts et al. 1996; Ogilvie \& Livio 2001). The ratio between this effective viscosity and resistivity (the nominal Prandtl number), would then determine whether the flux mostly moves inward with the mass, or instead diffuses outward relative to the mass fast enough to avoid much net inward motion.

This approach rests upon two little-examined assumptions: First, that the overall flow can be regarded as if it were laminar and time-steady, and second, that the behavior of the underlying turbulence can be reduced to parameters (an advection rate and a diffusion, i.e., resistivity, coefficient) whose values are independent of the magnetic field. With these assumptions, field motion in the disk body governs field motion far away from the disk, and one may describe the field evolution in the language of linear diffusion. Unfortunately, neither assumption applies to real accretion flows.

It is now well-established that angular momentum transport in accretion disks is due to turbulent magnetic stresses driven by the MRI. Consequently, the magnetic field structure is neither laminar nor time-steady. Even in the corona, where the MRI per se does not occur, the same basic physics leads (as we have earlier discussed) to irregular field motions. There is therefore no direct connection between local field motion deep inside the disk (e.g., resistive diffusion) and the position of the corresponding field lines far away. Moreover, as we have emphasized, flux is a global concept, not a local one; the coronal mechanism, for example, could never be described by a local theory of this sort.

The second assumption, that the evolution of the field can be described in terms of simple resistive and viscous diffusion operating on a background field gradient is similarly problematic. Accretion is driven by nonlinear magnetic stresses; because the effective inflow velocity arises only from an average of a strongly fluctuating velocity field, it is non-local in both time and space. Similarly, the breakdown in ideal MHD happens primarily by driven reconnection, the rate of which depends on the structure and magnitude of both the magnetic and velocity fields. As a result, neither the mean rate at which magnetic field is carried from place to place nor any tendency for its structure to smooth is either independent of or linear 
in the field strength.

In fact, the formulation in terms of a competition between "viscous" and "resistive" diffusion breaks down in an even more fundamental way. Where there is net flux passing through the disk body, the very same MHD turbulence that transports angular momentum simultaneously decouples the flux from the matter through turbulence-driven reconnection. Field lines associated with flux passing through the equator develop bends that readily break off as closed loops. The matter moves inward with the closed loops while the flux stays in place. Thus, in dramatic contrast to the conventional prediction, rapid "viscous" accretion can, and apparently does, co-exist with largely stationary flux, while the "resistivity" that permits this decoupling has no particular impact on smoothing the field distribution.

Moreover, there is relatively little net flux passing through the disk because reconnection associated with the coronal mechanism detaches it efficiently. In other words, most of the flux motion occurs in an "end-run" that bypasses the disk altogether, so that it has rather little to do with any activity in the disk body, "viscous", "resistive", or other.

Local smoothing of field structures can occur, but it is questionable whether one can define an overall diffusion coefficient to describe its rate. For example, Guan \& Gammie (2009) have recently attempted to quantify magnetic diffusion in the context of MRI-driven MHD turbulence using shearing box simulations. They imposed a sinusoidally varying vertical field with an amplitude above the background due to the MRI-turbulent flow and observed the subsequent decrease in this mode's Fourier power. The peak vertical field amplitudes studied vary from $10-80 \%$ of the initial toroidal field strength. They found that the decay time for the imposed feature is several tens of $\Omega^{-1}$, with the decay rate an increasing function of the perturbation amplitude. These results indicate that local field gradients can be smoothed within the turbulent flow, but it is unclear to what degree the inferred average diffusion coefficient depends on the specific situation studied. In particular, in accretion disks, the net field, the part determining the flux, is, especially in the disk body, small in magnitude compared to the fluctuating turbulent field. When the dynamics are strongly nonlinear, spreading of structures in this small net field may proceed in a way very different from spreading of larger amplitude structures; in fact, the motion of the net field may have much more to do with the dynamics of the fluctuating field than to its own disposition.

For all these reasons, therefore, we see no useful way to describe the results of the present simulation in the traditional language of arbitrarily specified transport coefficients applied to gradients of the time-averaged magnetic field.

Two other concepts have recently been studied. One of these (Rothstein \& Lovelace 2008; Lovelace et al. 2009) in some respects is a blend of the "advection/diffusion" picture 
and the coronal mechanism. On the one hand, it uses the conventional formalism of fixed transport coefficients; on the other hand, it relies heavily on field line motions in the corona. These two approaches are united by supposing that the MRI and its resulting turbulence are suppressed in the corona, so that it suffers neither "turbulent viscosity" nor "turbulent resistivity". Consequently, coronal motion is dominated by vertical transport of angular momentum, in a magneto-centrifugal wind when the effective Prandtl number is large, and in a Poynting flux-dominated outflow otherwise. The result is that (for most parameters), the upper surface of the disk moves inward, carrying the footpoints of the large-scale flux with it, while the flow in the midplane is outward.

As we have previously discussed, the fundamental physical element of the MRI, the creation of substantial magnetic stresses by orbital shear, does operate within the corona, and strongly - this is the foundation of the coronal mechanism. Despite these coronal motions, this is not a significant source of angular momentum transport for the main accretion disk. Although the instantaneous amplitude of the magnetic $T_{\phi}^{\theta}$ stress is typically sizable, outside the funnel its sign fluctuates so that we see no significant net vertical transport of angular momentum from the disk. In addition, the net fluid radial velocity in the midplane, although small compared to its rms value, is inward. Nonetheless, our results are consistent with their suggestion that magnetic stresses carrying angular momentum outward through the corona can be effective in moving field lines inward. Because the mass density in the corona is so low, removing a comparatively small amount of angular momentum can lead to large-scale field motions. Because the subsequent motions are not dominated by turbulence, flux freezing remains effective within the coronal plasma.

In the second of the two recent suggestions for how flux moves in accretion flows, Spruit \& Uzdensky (2005) propose that the flux motion is not controlled by a simple gradient, but rather by the dynamics of intermittent field bundles. These authors suggest that magnetized patches could accrete relatively rapidly from angular momentum losses through a wind. Further, if such fields were strong enough to suppress the MRI, then turbulence, and subsequently magnetic reconnection, would be greatly reduced for those patches. While such magnetized patches would not themselves lead to a significant net magnetic flux passing through the disk per se, they could accumulate at the central black hole until the field strength itself suppressed further accretion. Such a scenario is similar to that of the magnetically arrested accretion model of Narayan et al. (2003). Strong nonaxisymmetric field structures appear in a simulation of Igumenshchev (2008) where a strong field is continually injected at the outer boundary, providing some support for the Spruit \& Uzdensky (2005) concept. In our simulation, we do see nonaxisymmetric variations in the vertical flux throughout the disk, but not in the form of local pockets of intense vertical flux, nor do we see either any significant disk wind or any interruption of the accretion flow due to 
"magnetic arrest." Nonetheless, we agree with Spruit \& Uzdensky (2005) in emphasizing the intermittency of the flow and the potential importance of nonaxisymmetries.

The study carried out here is, of course, only a first step, and the results presented here point to several avenues of investigation with future numerical experiments. For example, simulations that continue for longer times and begin with net field at larger radii can better explore the efficacy of the coronal mechanism. In particular longer simulations with more available net field are required to see if the field strength in the funnel levels off or continues to build over time. Simulations in the Kerr metric with non-zero spin parameters could also study directly how magnetic flux evolution and the coronal mechanism relate to jet launching and collimation. The requirements of three spatial dimensions and increased spatial resolution will continue to make these investigations challenging.

This work was supported by NSF grant PHY-0205155 and NASA grant NNX09AD14G (JFH), and by NSF grant AST-0507455 (JHK). We thank Sean Matt, Jim Pringle, Chris Reynolds, Charles Gammie and Scott Noble for useful discussions. We acknowledge JeanPierre De Villiers for improvements to the algorithms used in the GRMHD code. The simulations described were carried out on the Teragrid Ranger system at TACC, supported by the National Science Foundation.

\section{REFERENCES}

Balbus, S. A., \& Hawley, J. F. 1991, ApJ, 376, 214

—. 1998, Reviews of Modern Physics, 70, 1

Beckwith, K., Hawley, J. F., \& Krolik, J. H. 2008a, ApJ, 678, 1180, arXiv:0709.3833

- 2008b, MNRAS, 390, 21, 0801.2974

Blandford, R. D., \& Payne, D. G. 1982, MNRAS, 199, 883

Blandford, R. D., \& Znajek, R. L. 1977, MNRAS, 179, 433

De Villiers, J., \& Hawley, J. F. 2003, ApJ, 589, 458

De Villiers, J., Hawley, J. F., \& Krolik, J. H. 2003, ApJ, 599, 1238

De Villiers, J., Hawley, J. F., Krolik, J. H., \& Hirose, S. 2005, ApJ, 620, 878

De Villiers, J.-P. 2006, ArXiv Astrophysics e-prints, astro-ph, astro-ph/0605744 
Evans, C. R., \& Hawley, J. F. 1988, ApJ, 332, 659

Ghosh, P., \& Abramowicz, M. A. 1997, MNRAS, 292, 887

Guan, X., \& Gammie, C. F. 2009, ArXiv e-prints, 0903.3757

Hawley, J. F., \& Krolik, J. H. 2006, ApJ, 641, 103, astro-ph/0512227

Heyvaerts, J., Priest, E. R., \& Bardou, A. 1996, ApJ, 473, 403

Hirose, S., Krolik, J. H., De Villiers, J., \& Hawley, J. F. 2004, ApJ, 606, 1083

Igumenshchev, I. V. 2008, ApJ, 677, 317, arXiv:0711.4391

Koide, S., Shibata, K., \& Kudoh, T. 1999, ApJ, 522, 727

Krolik, J. H., Hawley, J. F., \& Hirose, S. 2005, ApJ, 622, 1008

Livio, M. 2000, in American Institute of Physics Conference Series, Vol. 522, American Institute of Physics Conference Series, ed. S. S. Holt \& W. W. Zhang, 275-297

Livio, M., Ogilvie, G. I., \& Pringle, J. E. 1999, ApJ, 512, 100, arXiv:astro-ph/9809093

Lovelace, R. V. E., Rothstein, D. M., \& Bisnovatyi-Kogan, G. S. 2009, ArXiv e-prints, 0906.0345

Lubow, S. H., Papaloizou, J. C. B., \& Pringle, J. E. 1994, MNRAS, 267, 235

MacDonald, D., \& Thorne, K. S. 1982, MNRAS, 198, 345

Matsumoto, R., Uchida, Y., Hirose, S., Shibata, K., Hayashi, M. R., Ferrari, A., Bodo, G., \& Norman, C. 1996, ApJ, 461, 115

McKinney, J. C., \& Blandford, R. D. 2009, MNRAS, 394, L126, 0812.1060

McKinney, J. C., \& Gammie, C. F. 2004, ApJ, 611, 977, astro-ph/0404512

Narayan, R., Igumenshchev, I. V., \& Abramowicz, M. A. 2003, PASJ, 55, L69, arXiv:astro$\mathrm{ph} / 0305029$

Noble, S. C., Krolik, J. H., \& Hawley, J. F. 2008, ArXiv e-prints, 0808.3140

Ogilvie, G. I., \& Livio, M. 2001, ApJ, 553, 158, arXiv:astro-ph/0007474

Pudritz, R. E., Ouyed, R., Fendt, C., \& Brandenburg, A. 2007, in Protostars and Planets V, ed. B. Reipurth, D. Jewitt, \& K. Keil, 277-294 
Rees, M. J., Phinney, E. S., Begelman, M. C., \& Blandford, R. D. 1982, Nature, 295, 17

Reynolds, C. S., Garofalo, D., \& Begelman, M. C. 2006, ApJ, 651, 1023, arXiv:astro$\mathrm{ph} / 0607381$

Rothstein, D. M., \& Lovelace, R. V. E. 2008, ApJ, 677, 1221, arXiv:0801.2158

Schnittman, J. D., Krolik, J. H., \& Hawley, J. F. 2006, ApJ, 651, 1031, arXiv:astro$\mathrm{ph} / 0606615$

Spruit, H. C., \& Uzdensky, D. A. 2005, ApJ, 629, 960, arXiv:astro-ph/0504429

Thorne, K. S., Price, R. H., \& MacDonald, D. A. 1986, Black holes: The membrane paradigm (Black Holes: The Membrane Paradigm)

Tout, C. A., \& Pringle, J. E. 1996, MNRAS, 281, 219

Uzdensky, D. A., \& Goodman, J. 2008, ApJ, 682, 608, 0803.0337

van Ballegooijen, A. A. 1989, in Astrophysics and Space Science Library, Vol. 156, Accretion Disks and Magnetic Fields in Astrophysics, ed. G. Belvedere, 99-106 

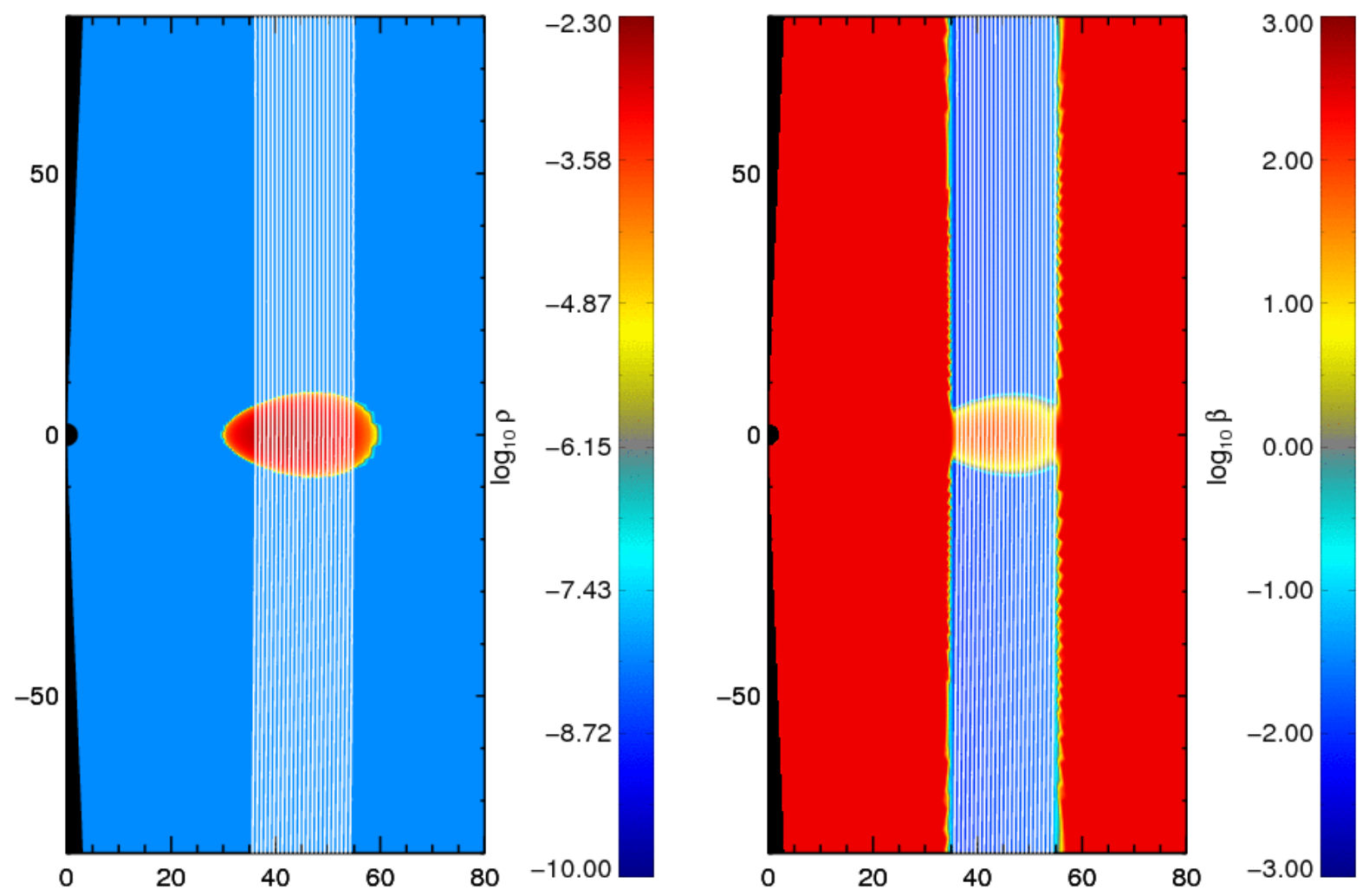

Fig. 1.- Initial torus and field configuration. White contours denote magnetic field lines, color contours density distribution (left panel) and the gas $\beta$ parameter (right panel). 

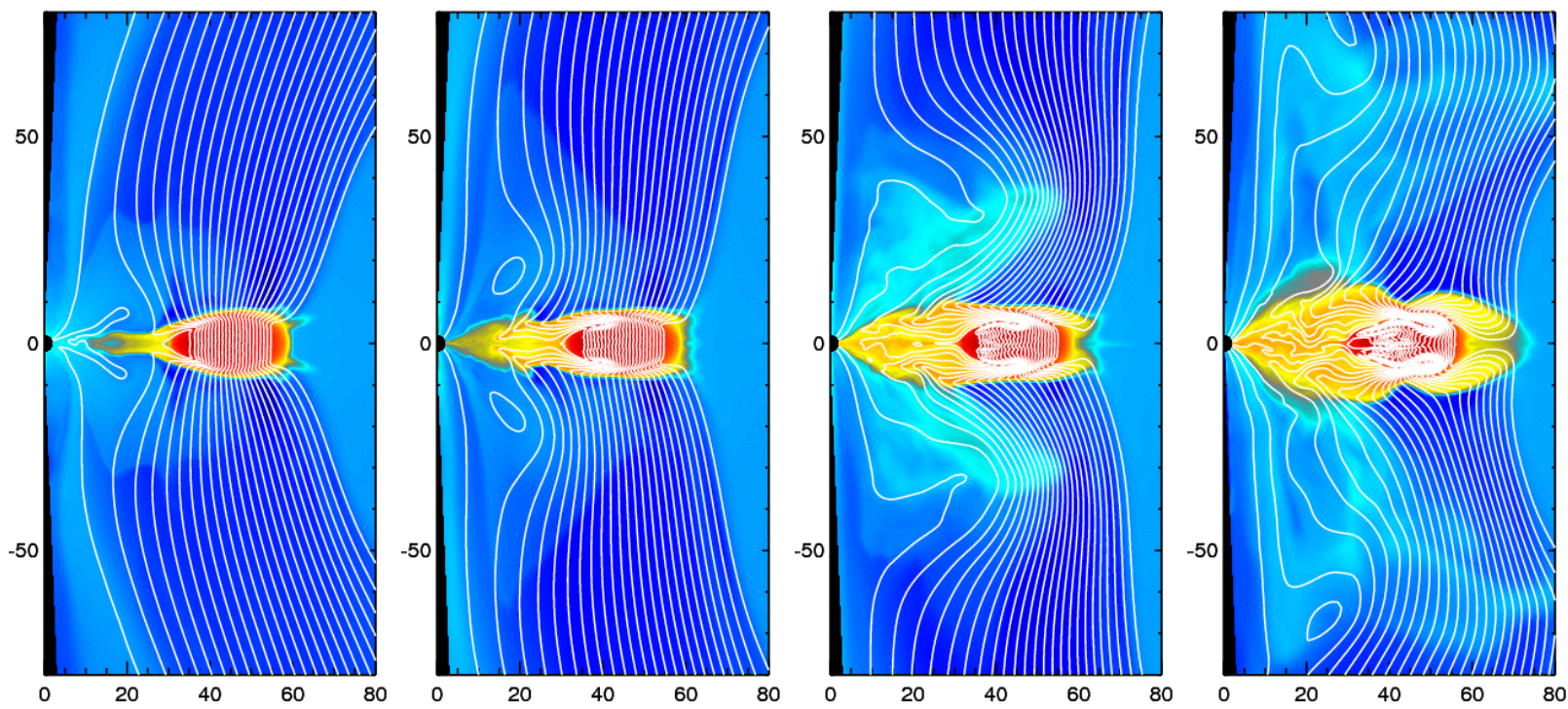

$\log _{10} \rho$

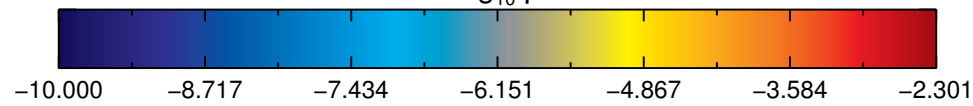

Fig. 2.- Initial evolution of the flow in simulation VD0m3d showing the advection of the vertical field from the initial location of the torus to the black hole. White contours are magnetic field lines, color contours show the density distribution. From left to right, the panels correspond to times $t=1000,1500,2000,2500 M$. The color scale is the same as in Figure 1. By $t=2500 M$, the funnel region has become strongly magnetic pressure dominated and two large scale channel modes have formed within the initial torus. 

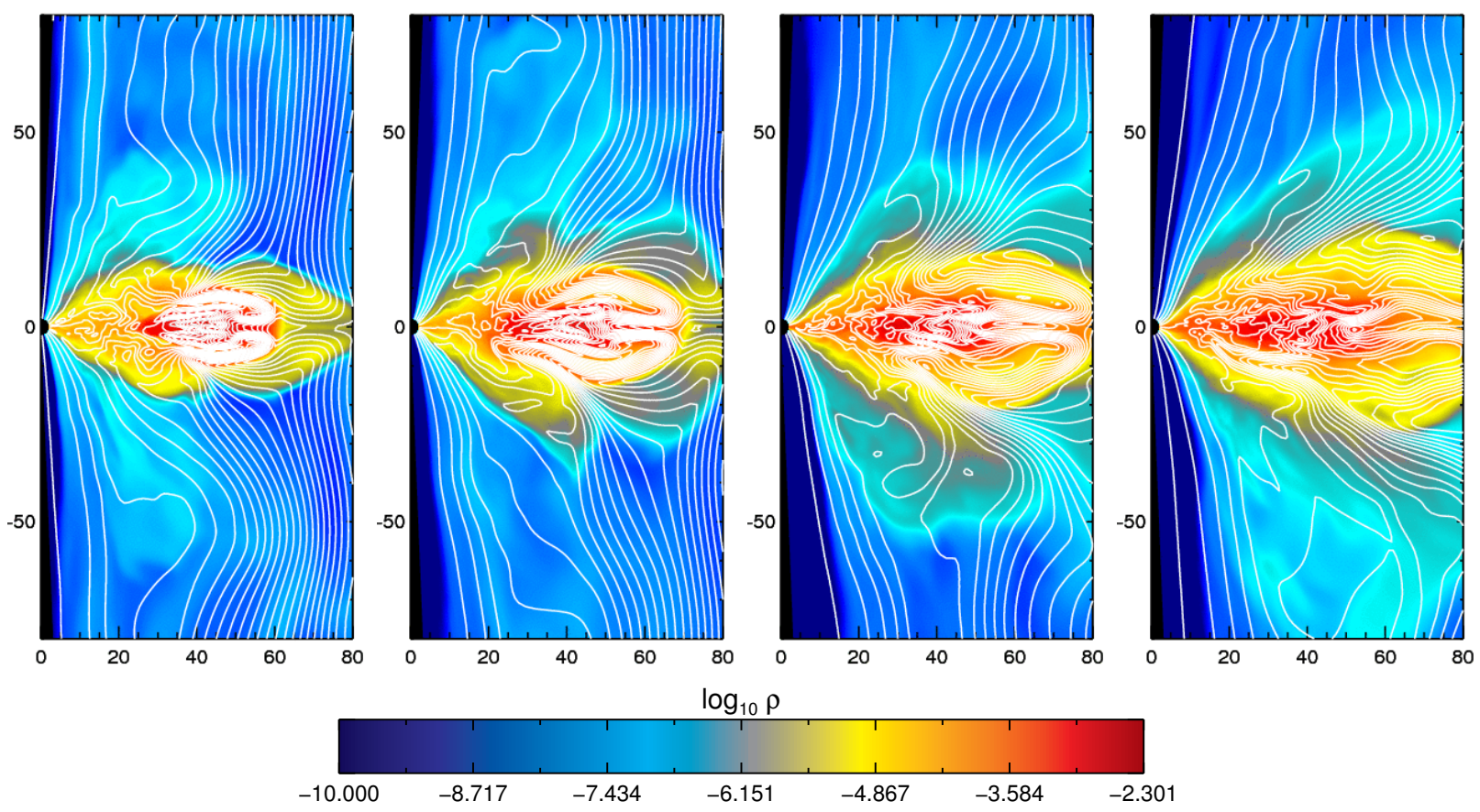

Fig. 3.- Growth and subsequent break up of the vertical field MRI channel mode in simulation VD0m3d. White contours are magnetic field lines, color contours show the density distribution. From left to right, the panels correspond to times $t=3000,3500,4000,4500 \mathrm{M}$. The color scale is the same as in Figure 1. By $t=4500 M$, the disk has become turbulent; large scale MRI modes persist within the corona. 


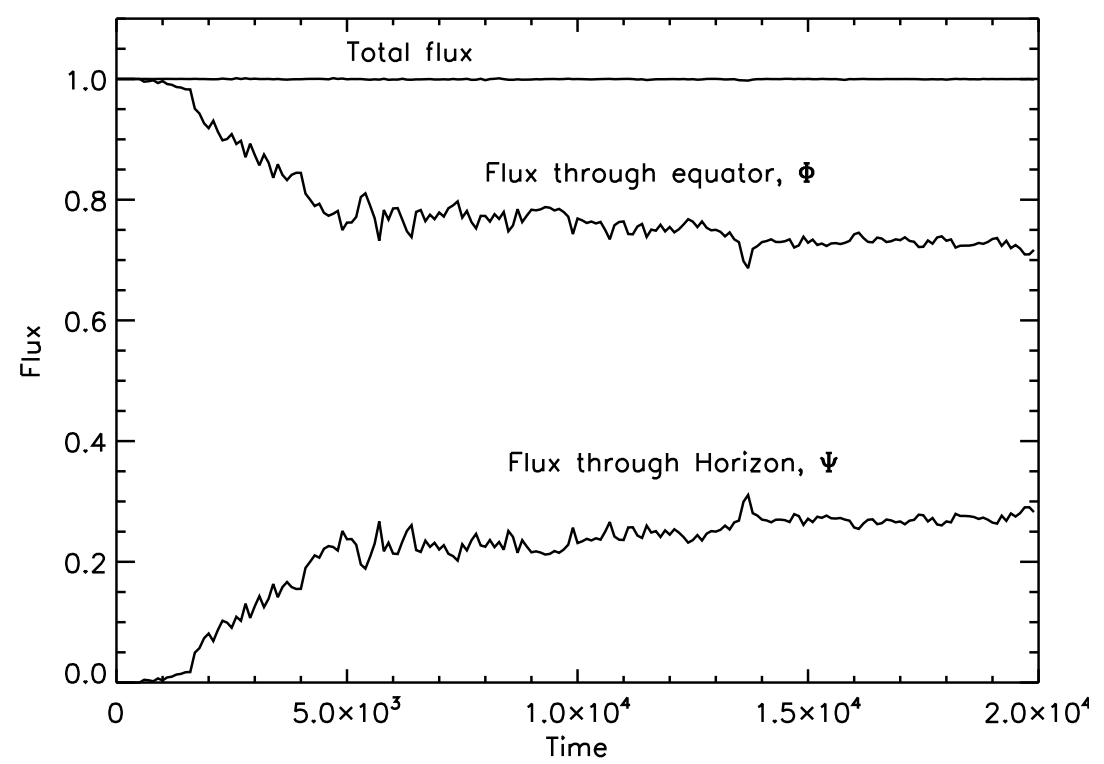

Fig. 4.- Total flux $A_{\phi}$ integrated from the axis along the horizon to the equator and then out along the equator to the outer boundary. The equatorial component, $\Phi$, and the horizon component, $\Psi$ are shown separately. The vertical scale is in units of the total initial flux. The total flux is unchanged through the evolution; losses in the equatorial flux are accounted for in net flux through the horizon. 

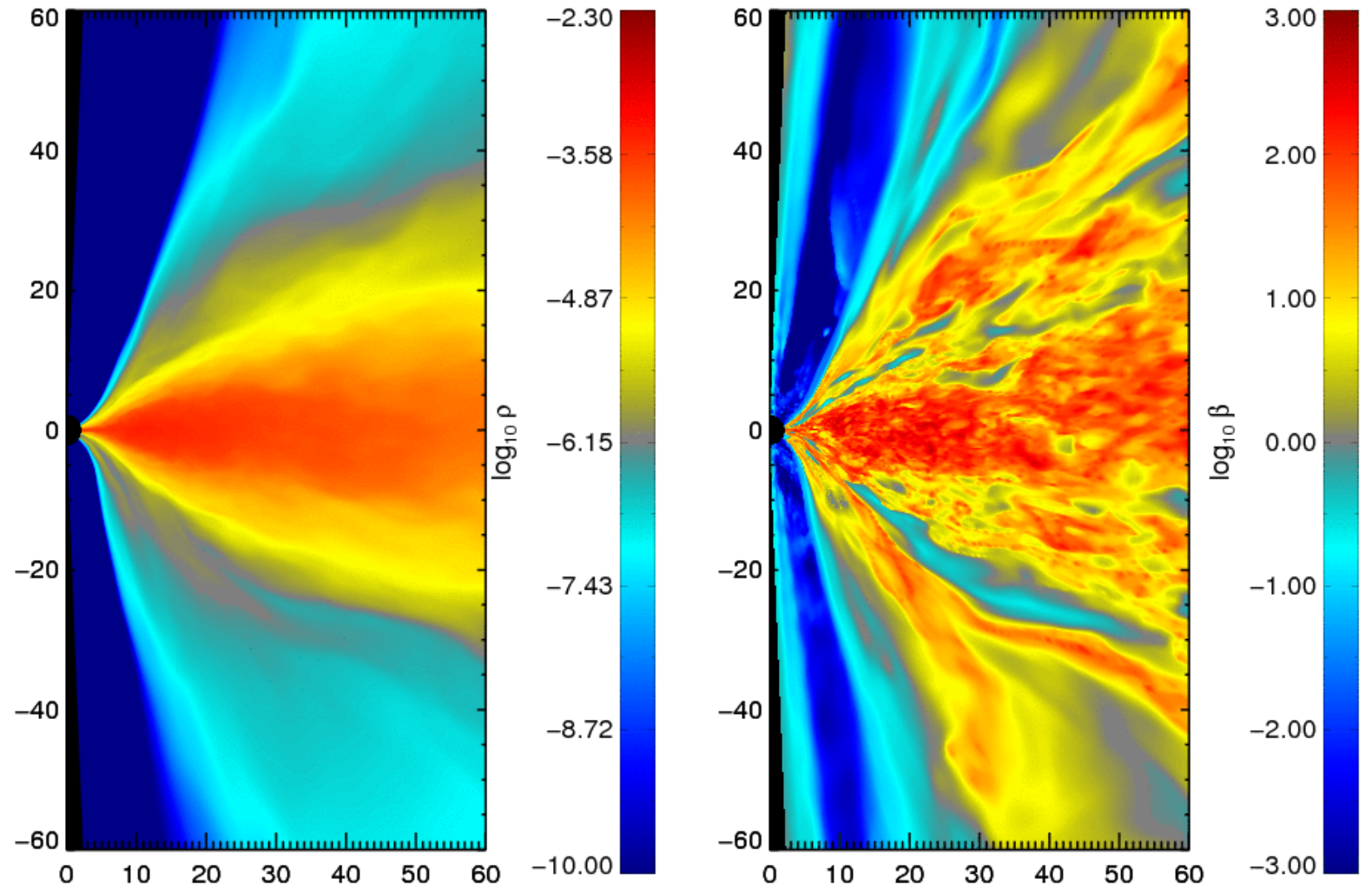

Fig. 5.- Azimuthally-averaged gas density (left panel) and plasma $\beta$ (right panel) at time $2 \times 10^{4} M$ in simulation VD0m3d. 


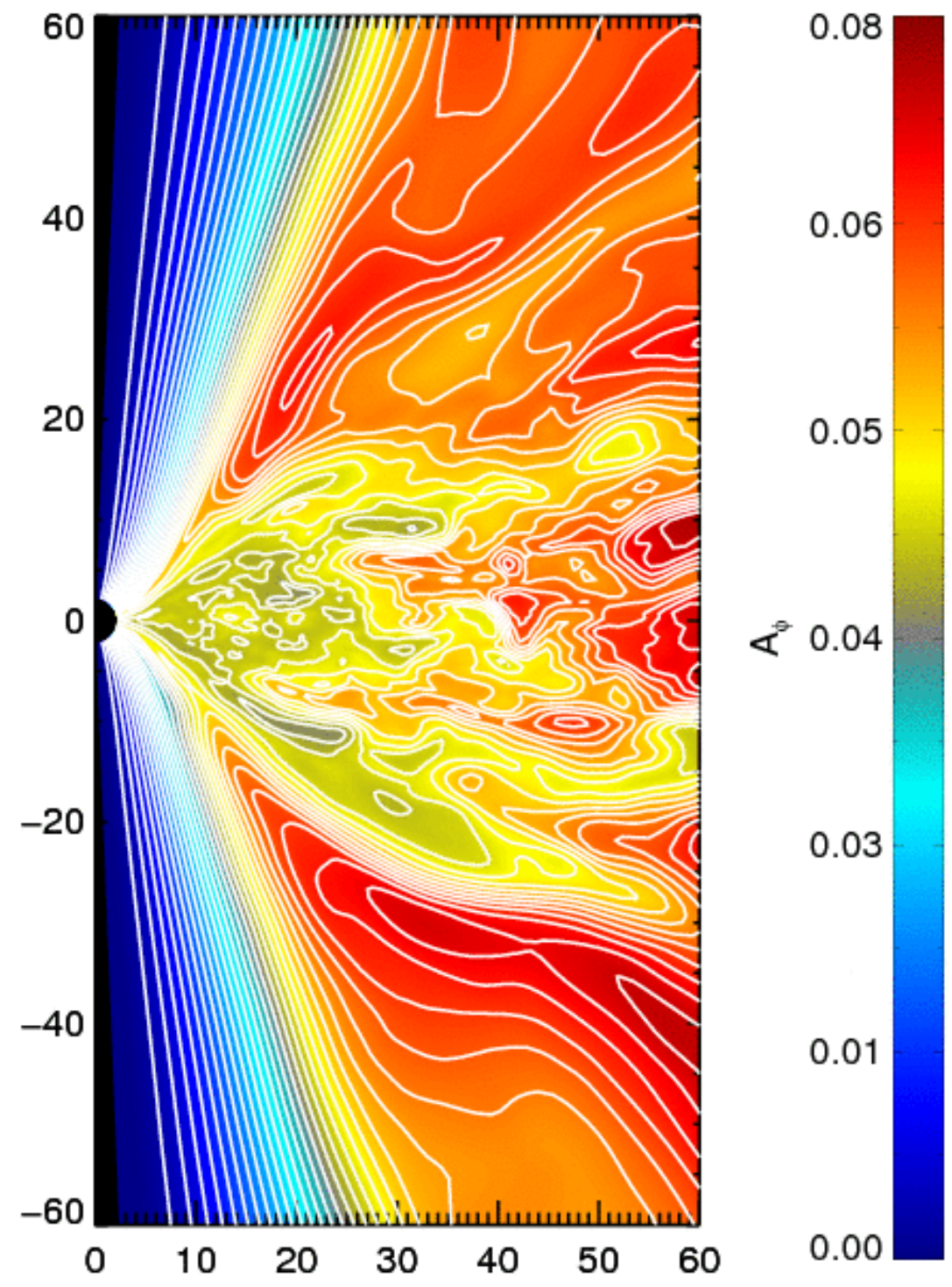

Fig. 6. - Contours of $A_{\phi}(r, \theta)$, displayed both in color and as poloidal field lines (isosurfaces of $\left.A_{\phi}(r, \theta)\right)$ at time $2 \times 10^{4} M$ in simulation VD0m3d. 

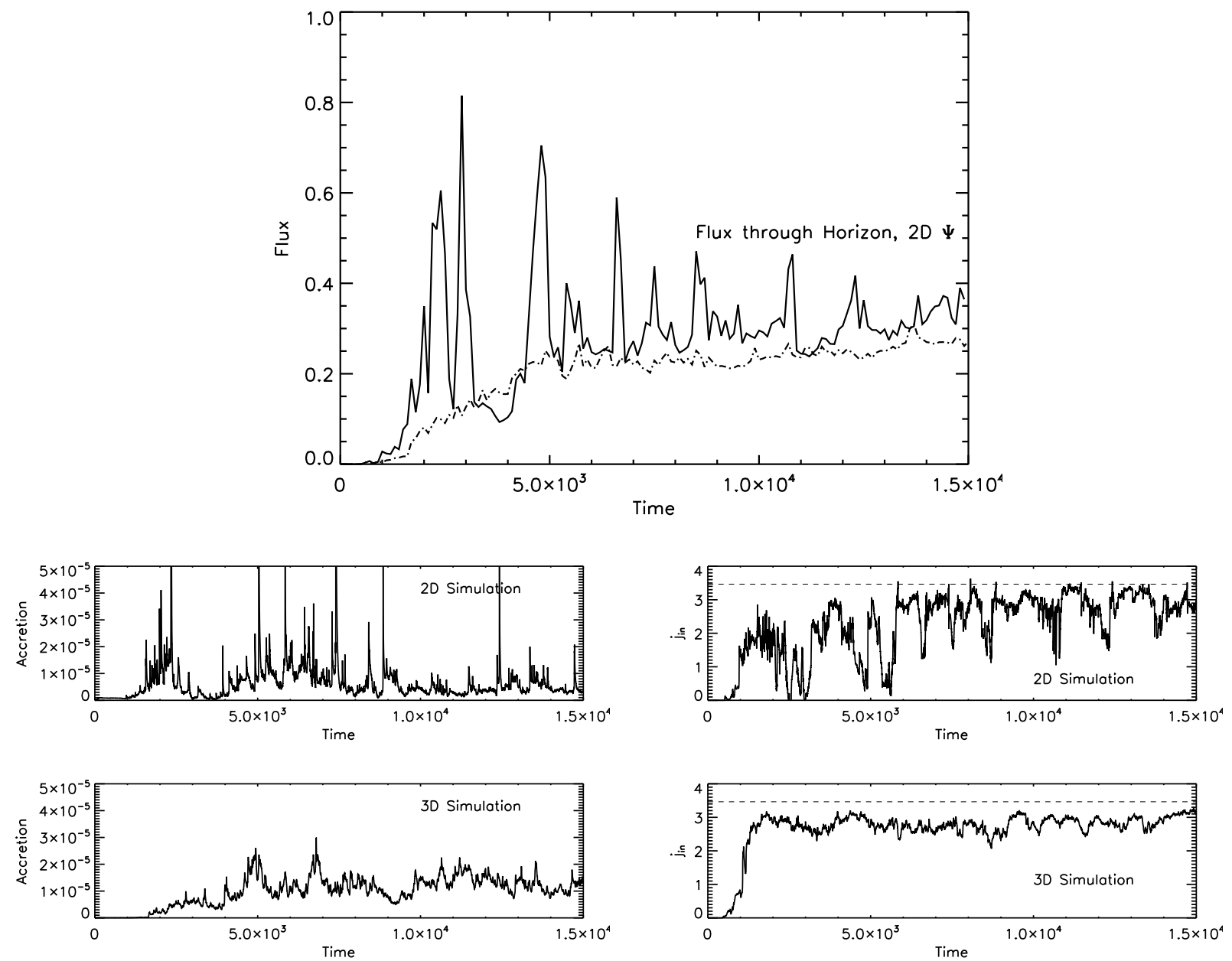

Fig. 7.- Comparison between results from the two-dimensional (VD0m2d) and threedimensional (VD0m3d) simulation. Top panel shows the flux through the horizon, $\Psi$ for VD0m2d (solid line) and VD0m3d (dashed line). The middle panel shows the net mass accretion rate through the horizon in units of the initial torus mass. The bottom panel shows the specific angular momentum carried into the hole as a function of time for simulations VD0m2d and VD0m3d. The strong coherent radial fields in the two-dimensional system create large fluctations in these quantities. 

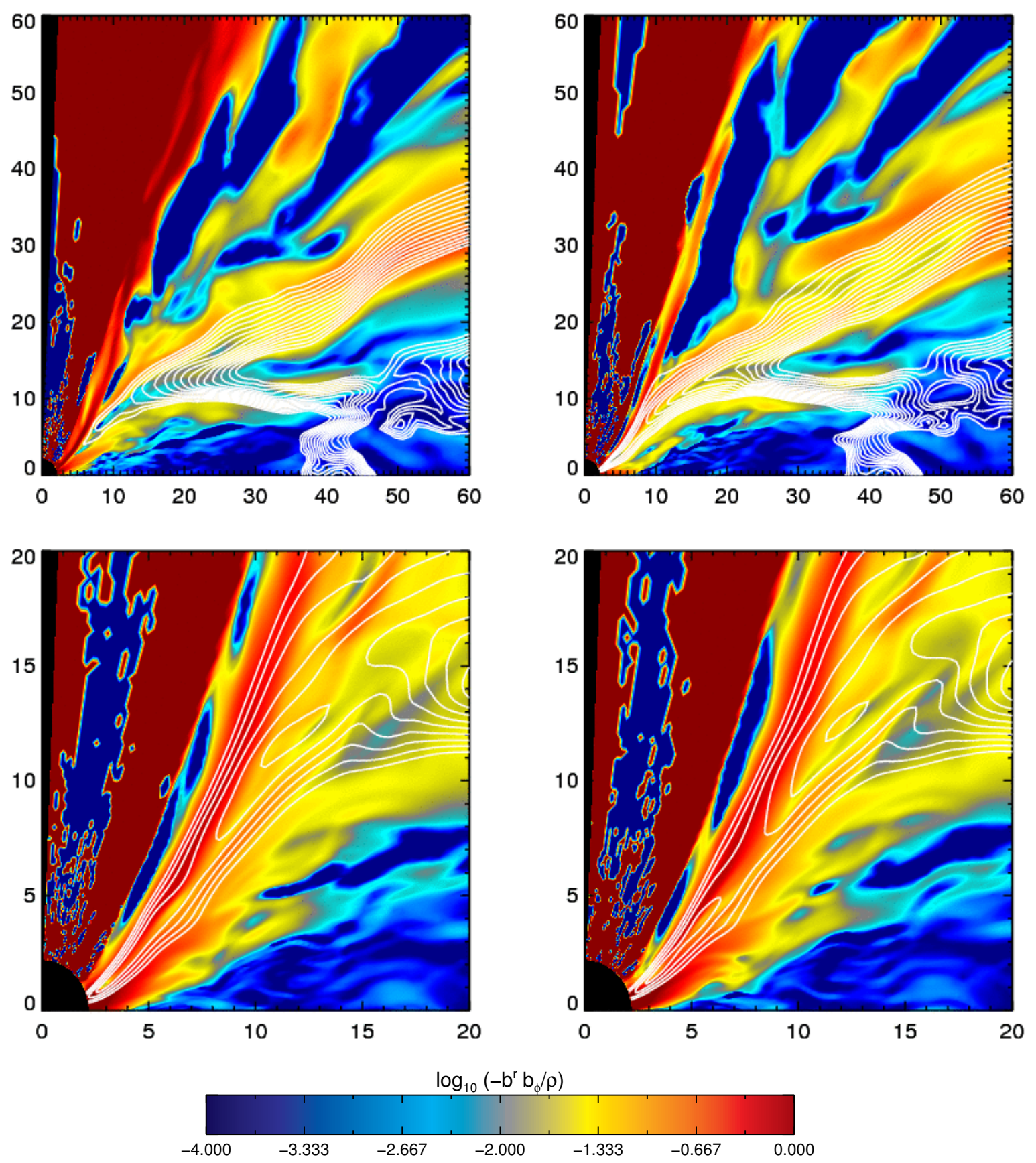

Fig. 8.- Evolution of a magnetic "hairpin" in the corona during the turbulent steady state. Color contours show the azimuthal average of stress per unit mass, $-b^{r} b_{\phi} / \rho$, and are overlaid with the poloidal field structure (white lines), which are kept fixed between the panels. From left to right, the top panels show times $t=6000$, and $6200 M$ (top row). The bottom row shows the near-hole region at times $t=6440$ and $6460 M$. During this time a reconnection event takes place within the field hairpin. 

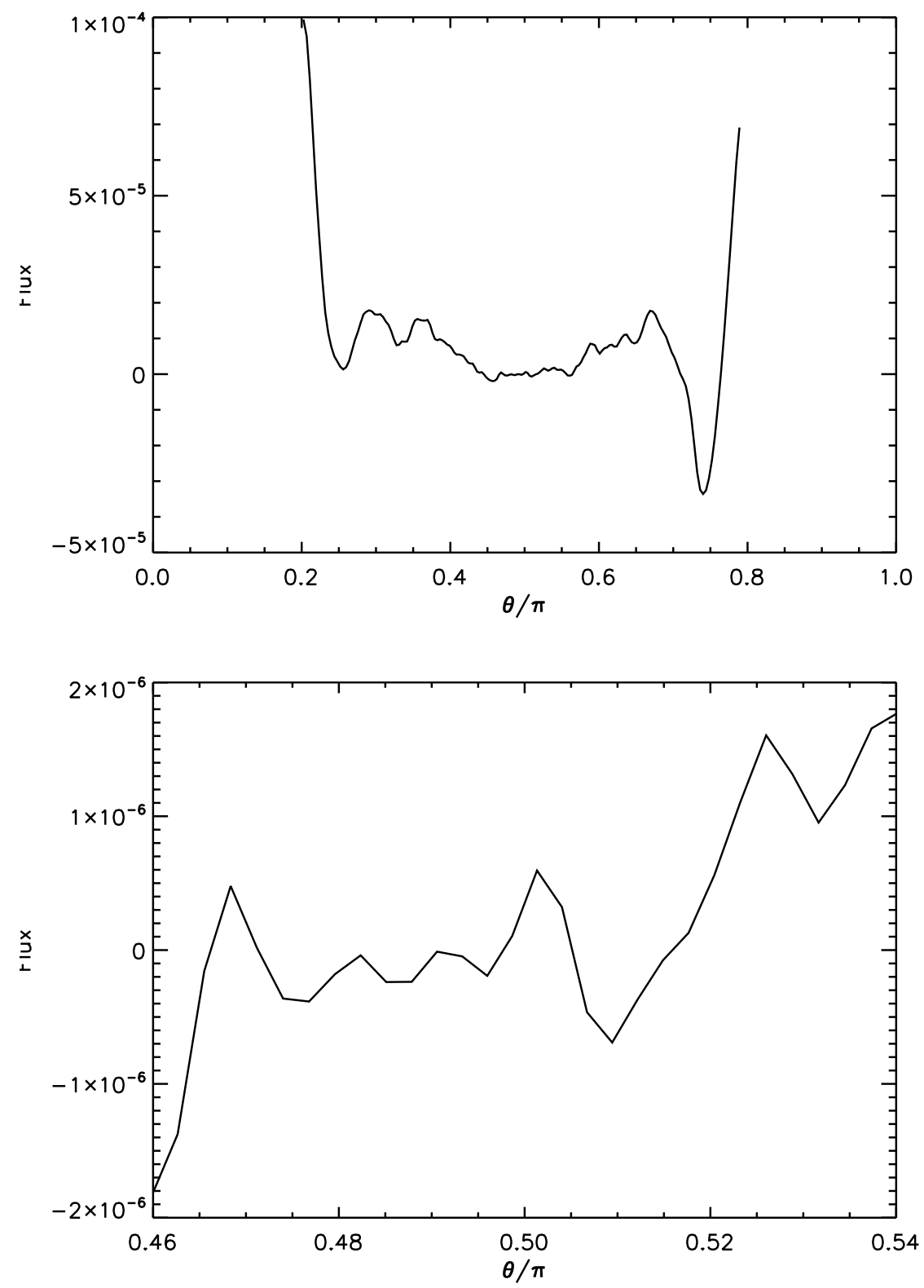

Fig. 9. - Azimuthal mean of $-V^{r} B^{\theta}(15 M)$, averaged over the time span $5000-1.5 \times 10^{4} M$ at $r=15 M$. Two $\theta$ ranges and scales are used: a scale appropriate for the corona and excluding only the funnel region (top), and only the disk proper plotted on a scale appropriate to the value there. 


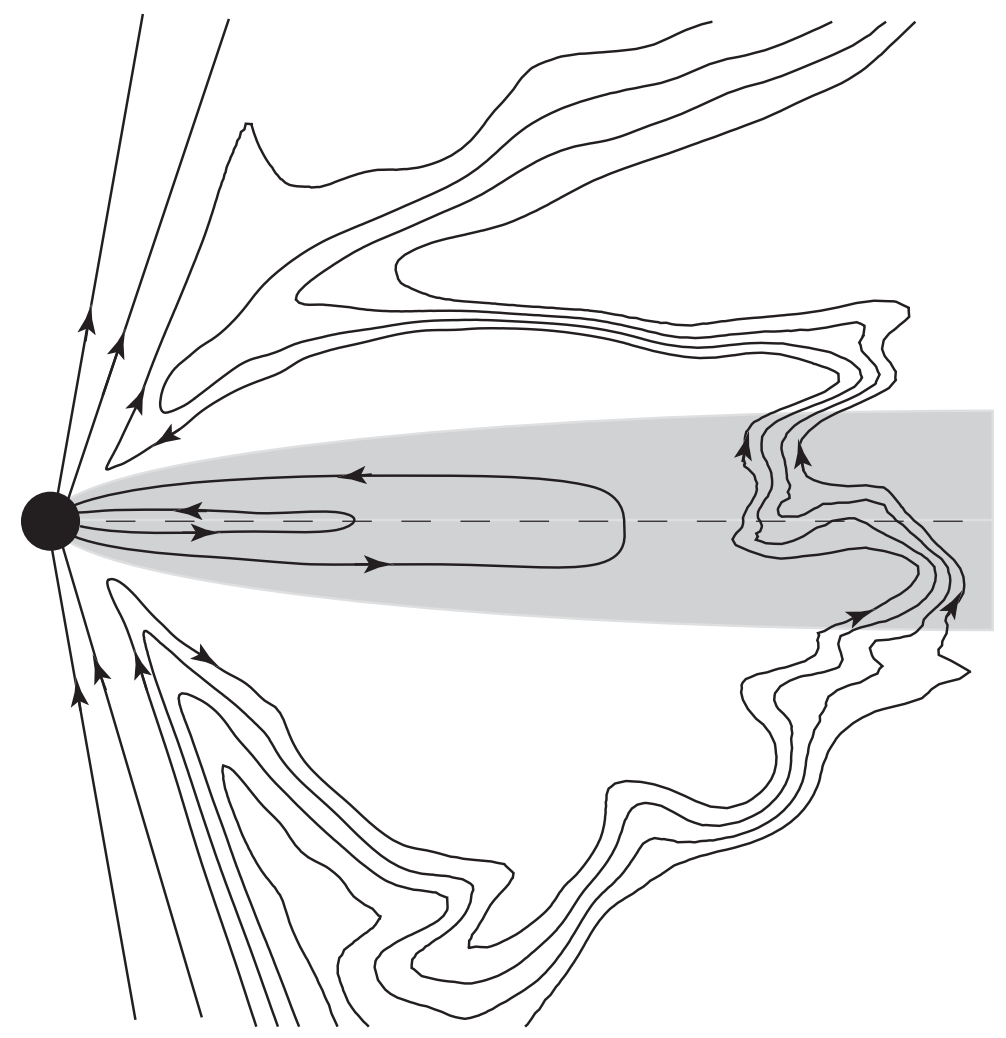

Fig. 10.- Schematic of the field line structure in the coronal mechanism. Field lines within the coronal are carried in toward the black hole, forming a hairpin-like structure. When the hairpin connects to the horizon flux is added to the funnel field and opposite-signed flux is added to the disk (shaded region). Reconnection across the equator (dashed line) allows this field to form loops that accrete. Accretion of those field loops results in an increase of the net horizon flux. 

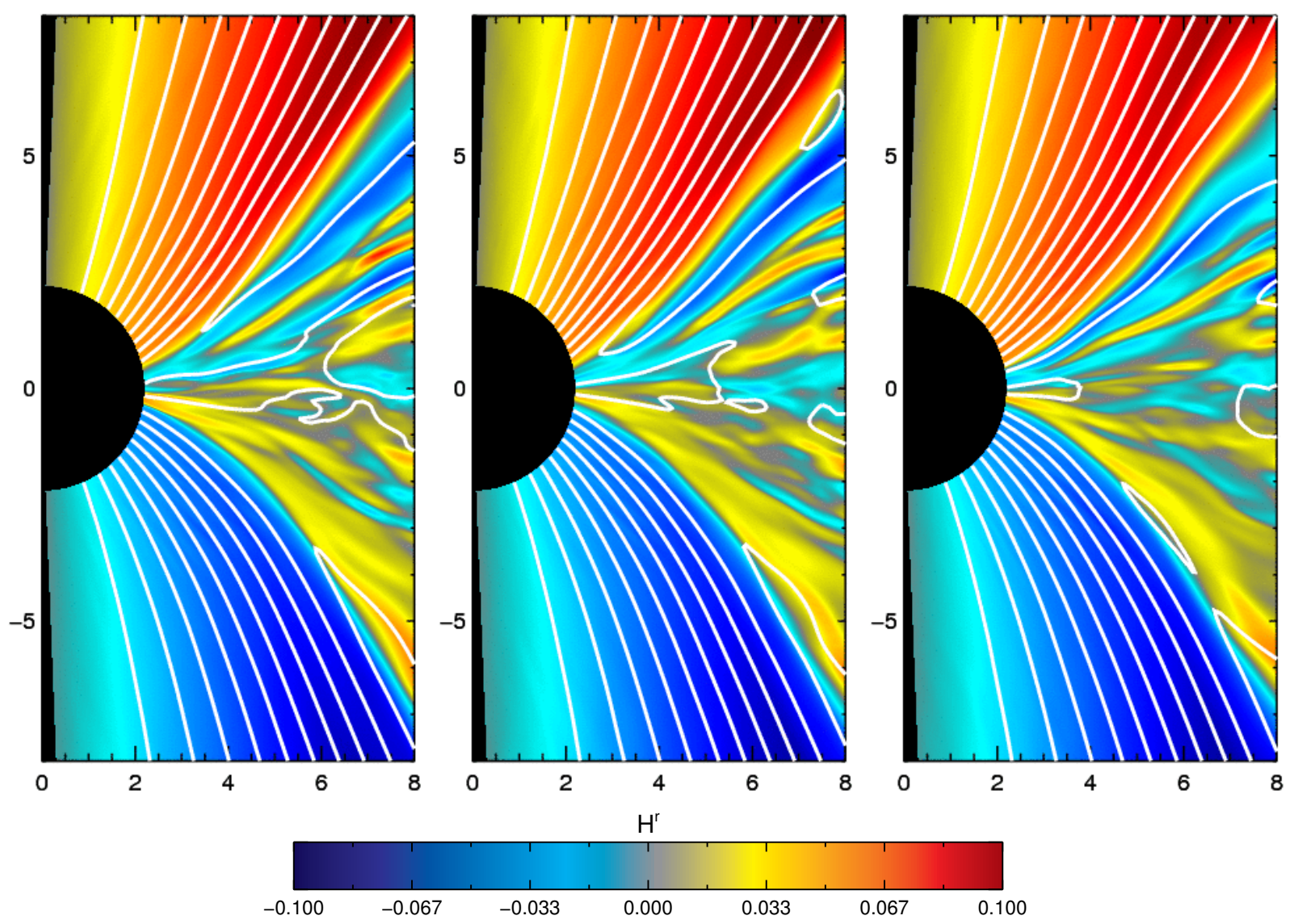

Fig. 11. - Reconnection event that occurs in the disk and leads to the rapid transport of poloidal magnetic flux from the outer disk to the black hole. Color contours show $\mathcal{B}^{r}$ and are overlaid with the poloidal field structure (white lines), which are kept fixed between the panels. From left to right the panels show $t=14600,14620$, and $14640 M$. 


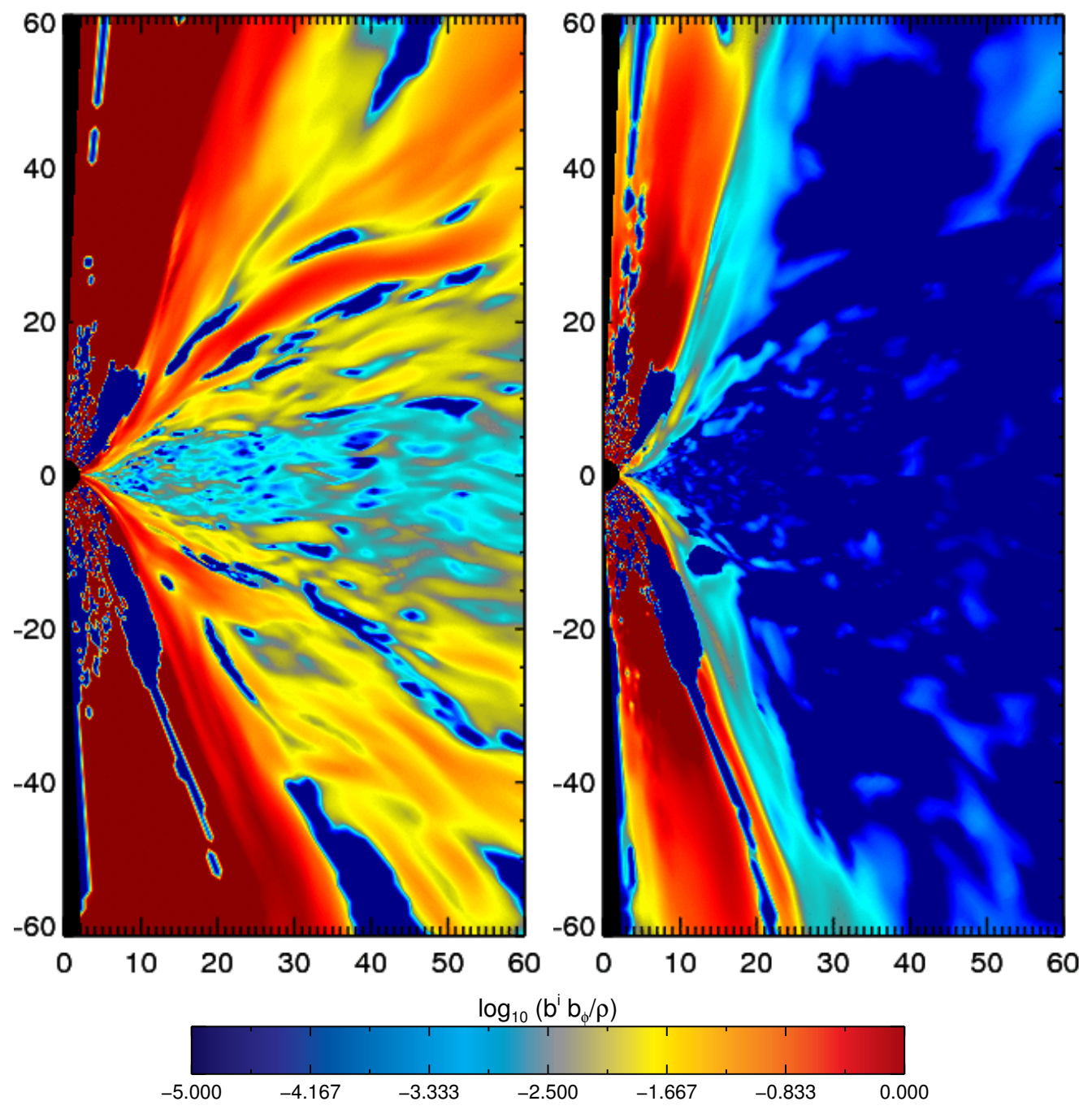

Fig. 12.- Azimuthal average of $-b^{r} b_{\phi} / \rho$ (left panel) and $-b^{\theta} b_{\phi} / \rho$ (right panel) at the same time as shown in Fig. 5. Regions with $-b^{i} b_{\phi} / \rho>1$ are colored dark red, regions with $-b^{i} b_{\phi} / \rho<10^{-5}$ (including $-b^{i} b_{\phi} / \rho<0$ ) are deep blue. The sign of $-b^{\theta} b_{\phi} / \rho$ is adjusted so that positive values correspond to electromagnetic angular momentum flux directed away from the disk. The azimuthal average of $-b^{\theta} b_{\phi}$ is much smaller than that of $-b^{r} b_{\phi}$ everywhere outside the funnel. 

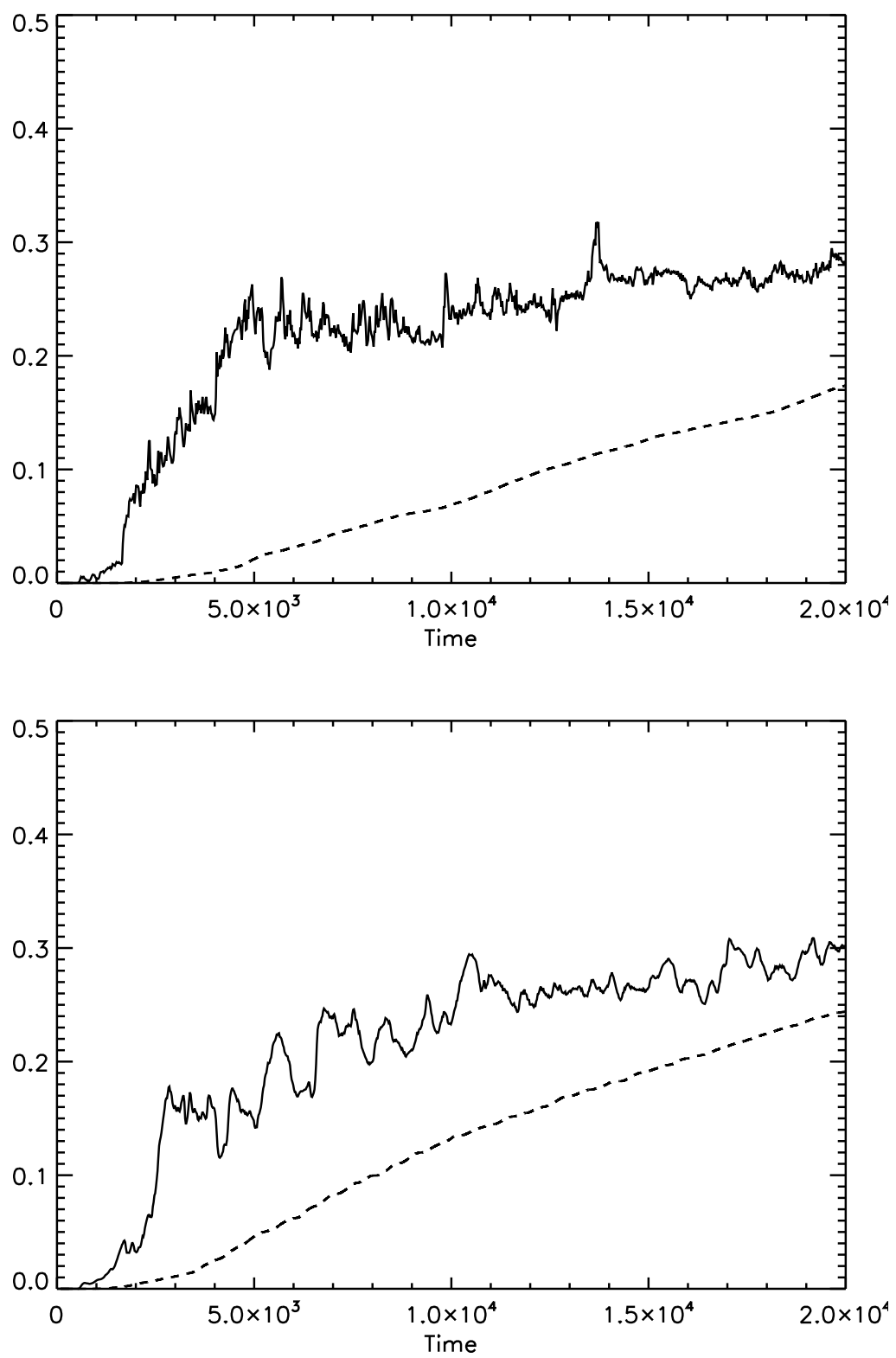

Fig. 13. - Fractional accretion of magnetic flux $\mathcal{A}$ (solid curve) and mass $\mathcal{M}$ (dashed curve) as functions of time. Because we stored shell-integrated mass accretion rates every $1 M$ in time, but the full 3 -d data required to compute the vector potential only every $10 M$, the latter curve has greater time resolution than the former. (Top) At $r=2.1 M$. (Bottom) At $r=20 M$. 


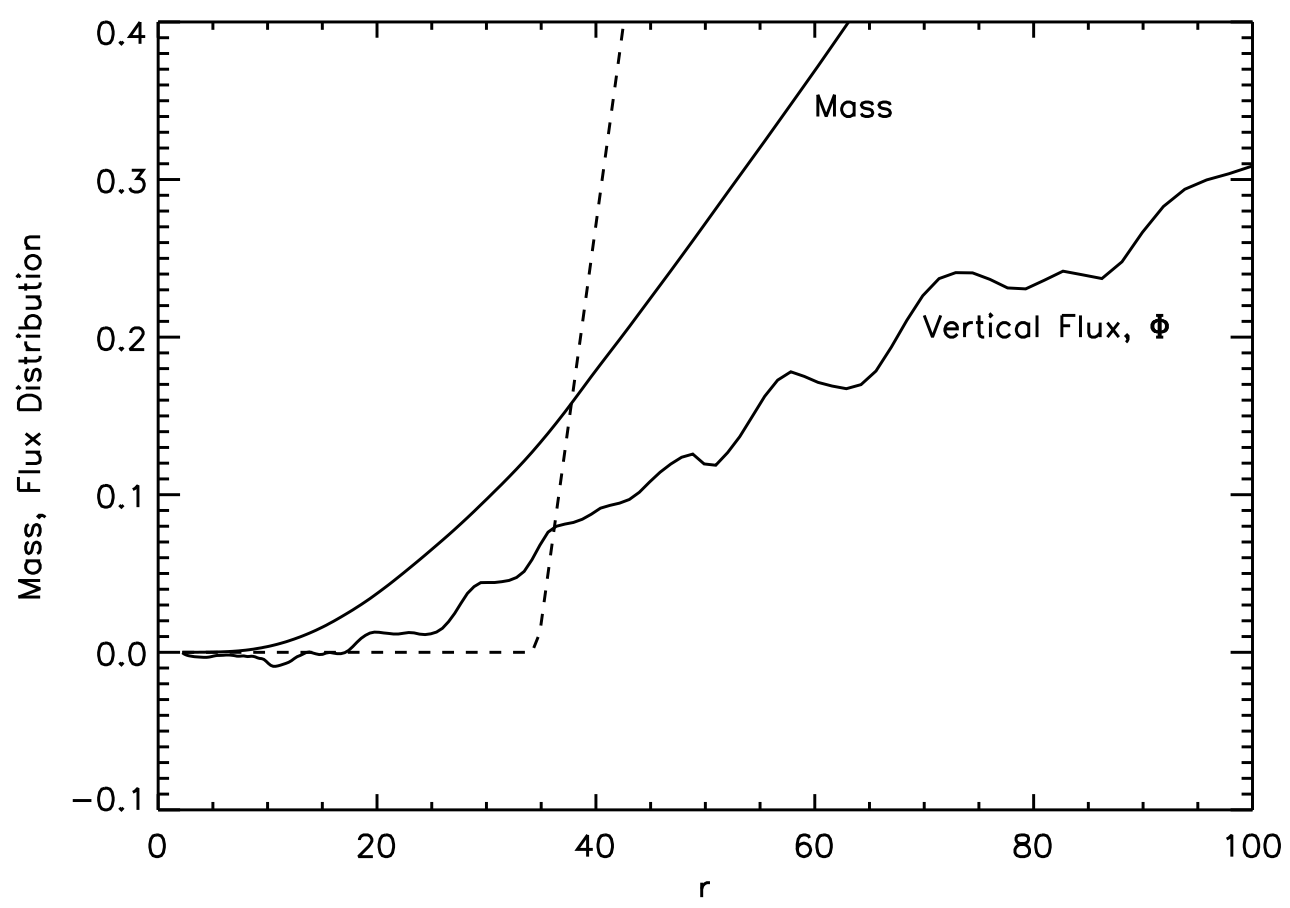

Fig. 14. - The net vertical flux through the equator $\Phi(r)$, integrated from the black hole horizon outward, averaged over the last $5000 \mathrm{M}$ of the simulation and normalized to the inital total value ("Vertical Flux, $\Phi ")$. The initial $\Phi$ distribution is also shown (dashed line). The net flux has spread through the disk over the course of the simulation. For comparison, the mass distribution $M(r)$ is shown (labeled "Mass") for the end time, $t=2 \times 10^{4} M$, likewise normalized to its initial total value. 


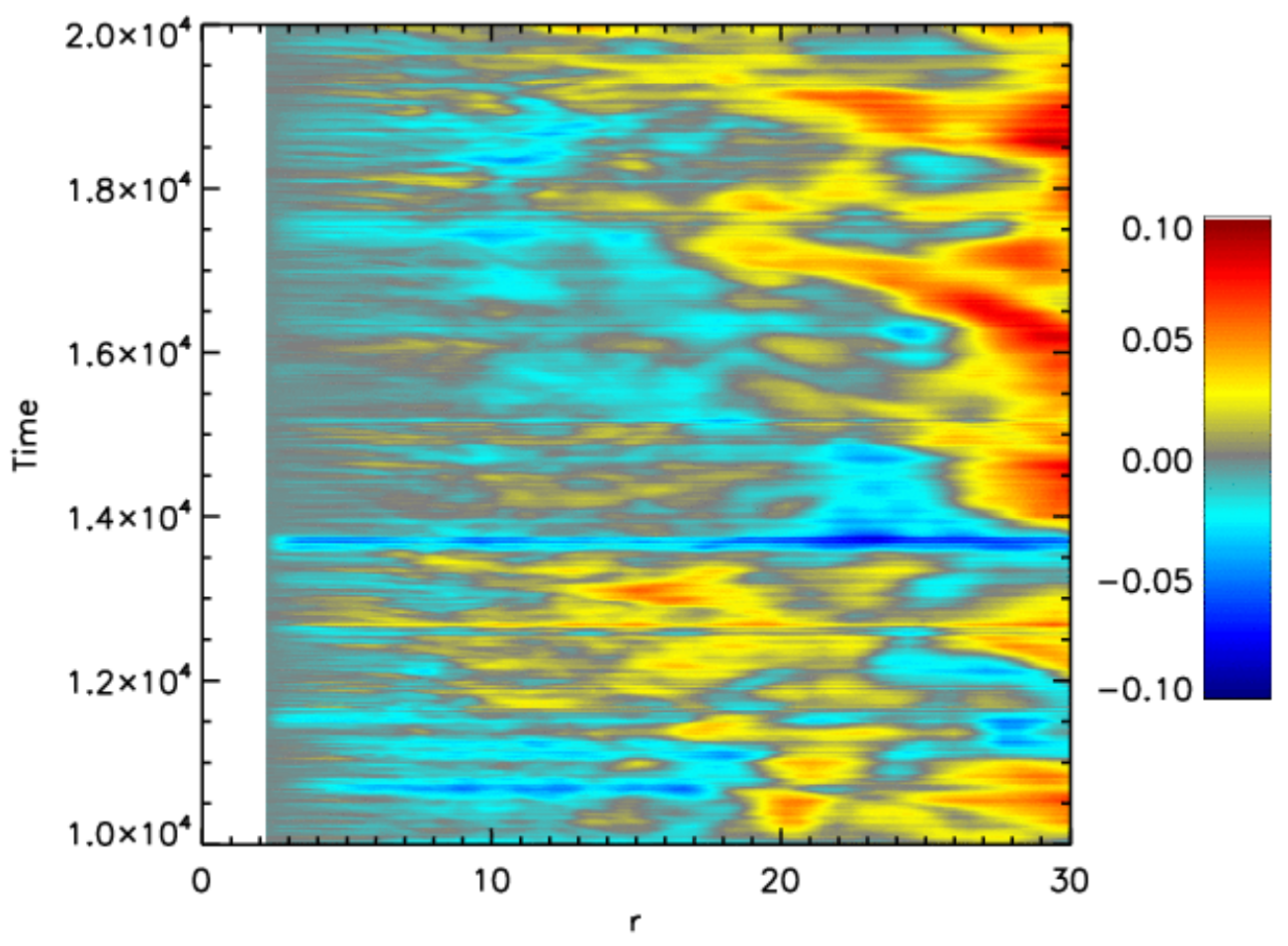

Fig. 15. - Space-time contours of the net vertical flux through the equator $\Phi(r)$, integrated from the black hole horizon outward for the inner disk during the second half of the simulation, normalized to the total initial flux. Never very large in magnitude, the integrated magnetic flux in this region fluctuates in sign. 


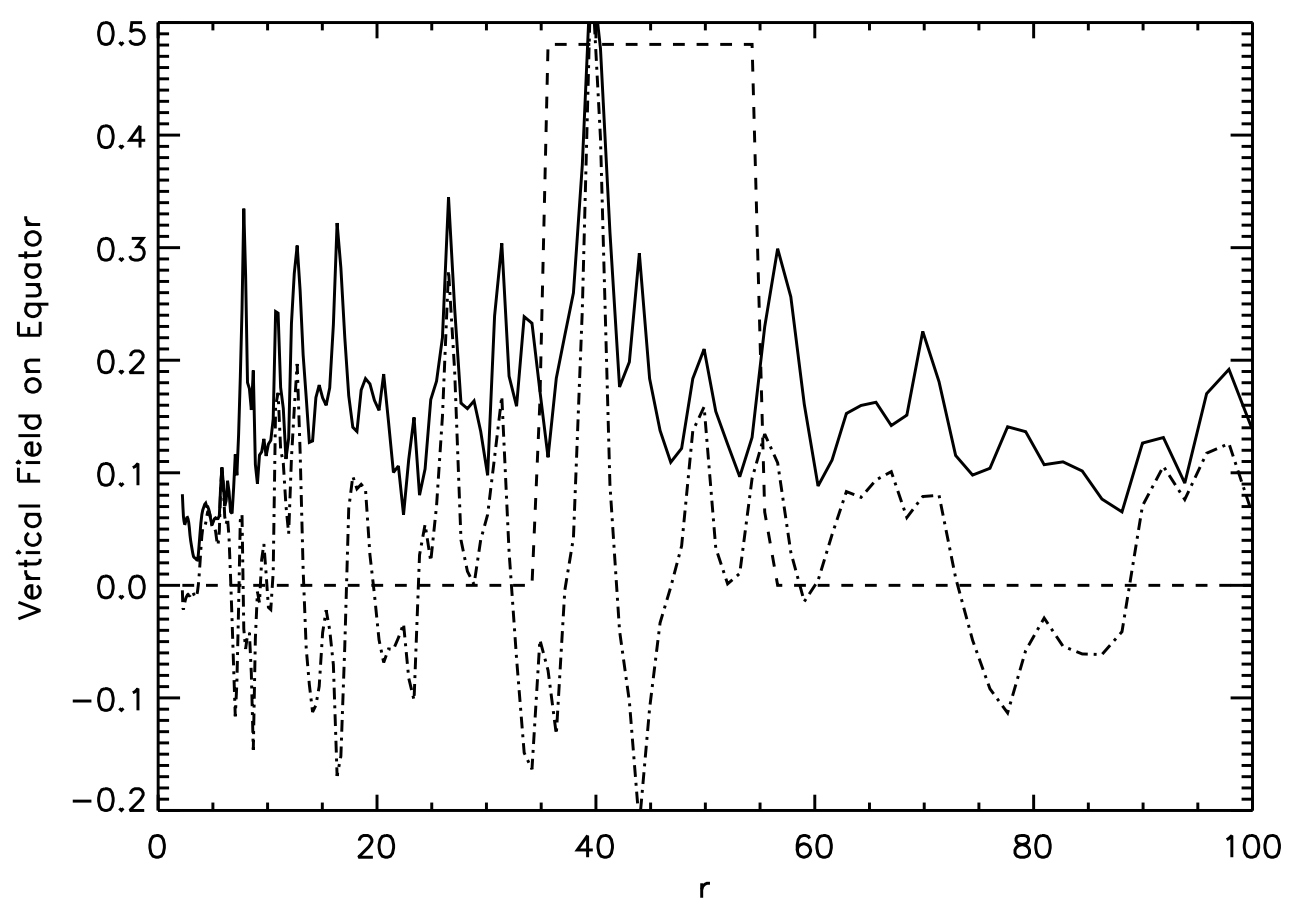

Fig. 16. - Azimuthal average of the absolute value of $\left|\mathcal{B}^{\theta}\right|$ (solid line) and the average of $\mathcal{B}^{\theta}$ (dot-dashed line) along the equatorial plane at $t=2 \times 10^{4} M$ (solid line) along with the initial $\mathcal{B}^{\theta}$ distribution (dashed line). The vertical field remains relatively strong but experiences fluctuations in sign throughout the disk body. The net flux is a small positive bias in the vertical field value. 


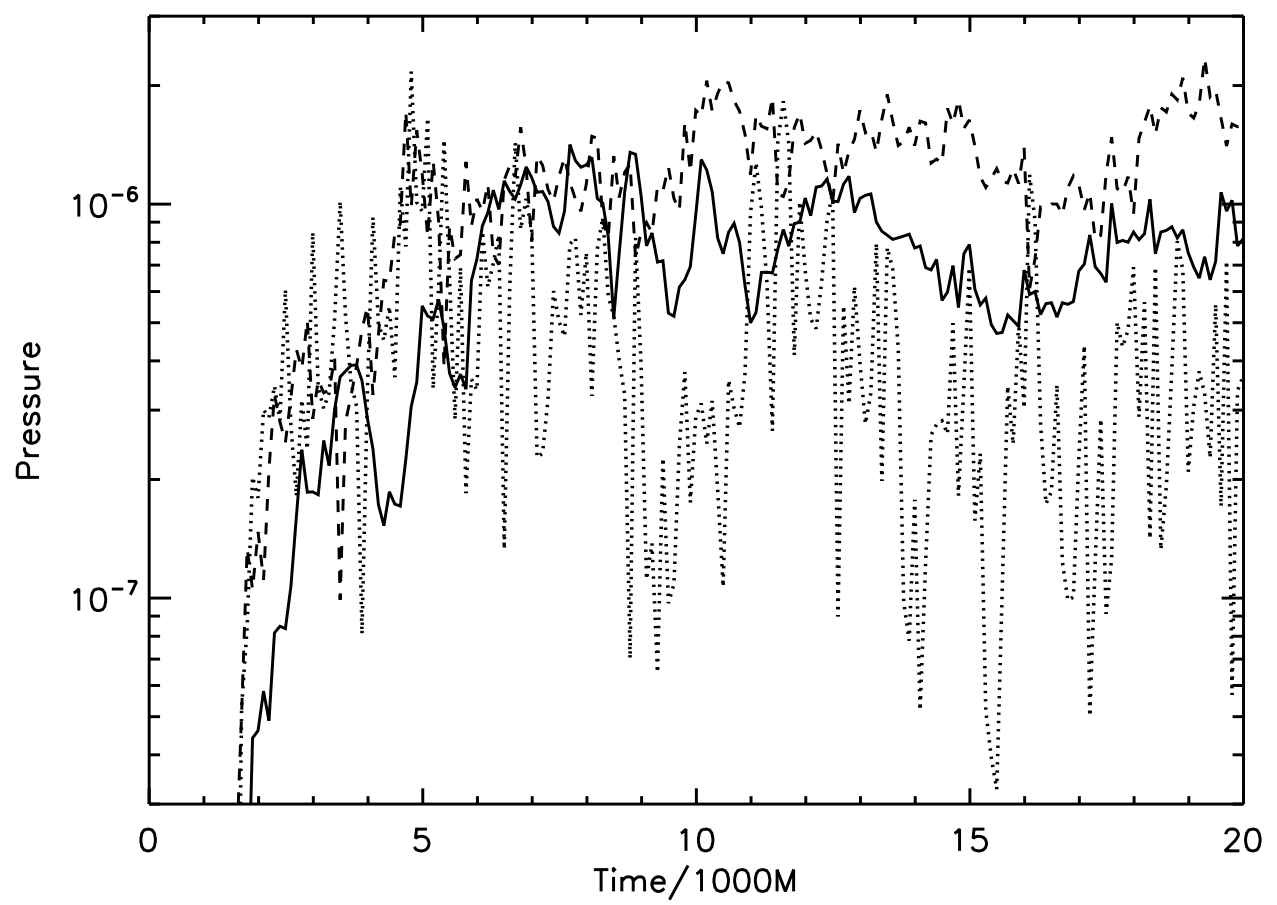

Fig. 17. - Azimuthally-averaged magnetic pressure as a function of time at two locations: inside the funnel near its base ( $r=6 M, \theta=0.044 \pi$; solid curve); and in the inner accretion flow ( $r=6 M, \theta=\pi / 2$; dotted curve), and azimuthally-averaged gas pressure in the inner accretion flow (also $r=6 M, \theta=\pi / 2$; dashed curve). 